\title{
Egoistic vs. altruistic beamforming in multi-cell systems with feedback and back-haul delays
}

\author{
Bruhtesfa E Godana ${ }^{1 *}$ and David Gesbert ${ }^{2}$
}

\begin{abstract}
Base station cooperation is an attractive technique to increase the spectral efficiency of multi-cell systems. One of the challenges in multi-cell systems is obtaining accurate channel state information (CSI) at the base station. One source of CSI inaccuracy is the delay incurred in obtaining CSI and exchanging it among base stations. The presence of delay is inevitable when CSI is exchanged over the back-haul. In addition, CSI is commonly obtained using limited feedback techniques which further contribute to its inaccuracy. In this paper, we re-visit the comparison between competitive (egoistic) and cooperative (altruistic) beamforming strategies in the presence of imperfect CSI. The impact of CSI inaccuracy due to delay and finite codebook size on the achieved sum rate is analyzed. Closed-form expressions for a lower bound and a first-order approximation of the average sum rates achieved by these beamforming strategies are derived. Using the closed-form expressions, a mode switching criterion is proposed to switch between competitive and cooperative beamforming based on the signal-to-noise ratio (SNR), delay, Doppler frequency and codebook size. It is shown that competitive beamforming is preferred in the limit of low SNR irrespective of the quality of CSI, while cooperative beamforming is preferred only at high SNR and low Doppler frequencies, confirming that base station cooperation is not always advantageous.
\end{abstract}

Keywords: Coordinated beamforming; Interference channel; Sum rate analysis; Back-haul delay; Limited feedback

\section{Introduction}

Multi-cell cooperation is known to improve the performance of cellular communication systems as compared to single-cell systems which treat the out-of-cell interference as noise [1-4]. Multi-cell cooperative transmission has also been proposed in upcoming standards such as LTE-Advanced to decrease inter-cell interference and improve data rate in a universal or partial frequency re-use framework [5,6].

Various levels of base station cooperation, ranging from full cooperation to simpler schemes involving only the sharing of channel state information (CSI), are considered for multi-cell systems [3]. While joint precoding, which requires CSI and user data sharing, provides the highest sum rate in an ideal CSI setting, it comes at a cost of large back-haul load and high overhead and complexity [7]. These factors, specially the capacity of the back-haul link, determine the level of cooperation and

\footnotetext{
*Correspondence: godana@iet.ntnu.no

${ }^{1}$ Department of Electronics and Telecommunications, Norwegian University

of Science and Technology, Trondheim 7491, Norway

Full list of author information is available at the end of the article
}

the transmission strategy in multi-cell systems [8-10]. Cooperative beamforming, which requires the exchange of CSI only, improves the sum rate with a reasonable back-haul load. In fact, the back-haul load for exchanging CSI is very small as compared to exchanging user data [9]. A comparison of cooperative (altruistic) and competitive (egoistic) beamforming strategies and the achievable rate regions in multiple-input single-output interference channel (MISO-IC) are studied in [11,12]. These beamforming strategies are distributed, non-iterative, and satisfy per base station power constraint. However, it is assumed that there is perfect CSI at the transmitter. In the presence of only statistical CSI, the achievable rate regions for a two-user MISO-IC are studied in [13].

In practice, base stations obtain delayed and quantized channel state information. CSI delay, which may be caused by propagation, signal processing or information routing, affects the performance of both single-cell systems [14] and multi-cell systems. Cooperative multi-cell systems

\section{包 Springer}

(c) 2013 Godana and Gesbert; licensee Springer. This is an Open Access article distributed under the terms of the Creative Commons Attribution License (http://creativecommons.org/licenses/by/2.0), which permits unrestricted use, distribution, and reproduction in any medium, provided the original work is properly cited. 
which exchange CSI over the back-haul network suffer the most since an extra back-haul latency can cause significant CSI outdating $[15,16]$. In frequency division duplex (FDD) systems, channel state information can only be delivered to the transmitter using limited feedback which further contributes to CSI inaccuracy $[17,18]$. Therefore, it is important to re-visit the performance comparison of egoistic and altruistic beamforming methods in the presence of quantized and delayed CSI. Based on the comparison, a mode switching criterion can be proposed in order to switch the transmission mode between single-cell (competitive) and multi-cell (cooperative) beamforming based on the signal-to-noise ratio (SNR) and quality of CSI. Beyond the mode switching method, the analysis also serves to gain a better understanding of which cooperative transmission method is the best depending on the feedback exchange properties. In particular, our intuition is that transmission mechanisms that rely on inter-base station CSI exchange can be powerful in an ideal CSIT (channel state information at the transmitter) setting but not as robust as simpler (matched filter based) strategies that can operate with local CSIT alone. The paper investigates this question by using closed-form analysis where possible.

In order to compare competitive and cooperative beamforming strategies, it is necessary to obtain the average sum rates in closed form. In [16], the average loss in sum rate due to limited feedback and delay using inter-cell interference cancellation is derived and used for feedback bit allocation. In [11], the average sum rate for a two-user MISO system is evaluated in closed form assuming availability of perfect CSI. The two-user analysis is extended in [13], in the availability of statistical CSI at the transmitter. The average sum rate achieved without delay, but with quantized CSI, is also derived in [19], though the derivations are only for two- and three-cell settings and expressed in terms of integrals. In general, the average closed-form sum rates achieved by egoistic and altruistic beamforming with quantized and delayed CSI had not been derived in the literature for a general multi-cell setting.

In this paper, the performance of egoistic and altruistic beamforming schemes is compared in the presence of CSI inaccuracy using the average sum rate as a metric. The main emphasis of the paper is to evaluate the effect of feedback delay and back-haul delay; nevertheless, the effect of limited feedback is also considered for completeness. Closed-form expressions for a lower bound and a first-order approximation of the average sum rates are obtained. Using these expressions, a mode switching criterion is obtained to switch between these beamforming schemes based on the SNR and quality of CSI. In this work, it is assumed that all base stations in a cluster use the same beamforming scheme; as a result, mode switching can be done in a decentralized fashion. Though it is possible to improve the sum rate marginally by using an adaptive scheme where base stations can adopt different schemes as discussed in [19], this method has some limitations. Firstly, the complexity of mode selection grows exponentially with the cluster size. Secondly, such a method assumes that each base station knows the scheme selected by the other base stations; however, this requires back-haul information exchange in practice, which causes further performance degradation in the presence of delay. Moreover, the performance gained by such an adaptive scheme can be rather gained through dynamic clustering and using a clusterwide beamforming scheme. Therefore, it is assumed in this paper that all base stations in a cluster use the same beamforming scheme. The paper has the following main contributions:

- Simplified closed-form expressions for a lower bound and a first-order approximation of the ergodic sum rates achieved by competitive and cooperative beamforming are derived. The validity of the lower bounds and approximations is demonstrated.

- A mode switching criterion is obtained to switch between these beamforming schemes based on the quality of CSI and the SNR. This helps to avoid the unnecessary load of CSI sharing in the case where cooperation is not useful.

The paper is organized as follows. In Section 2, a multicell MISO-IC and models representing the effect of delay, limited feedback, and user locations are outlined and discussed. In Section 3, the competitive and cooperative beamforming schemes used in this paper are reviewed. In Section 4, closed-form expressions for a lower bound and a first-order approximation of the average sum rates are derived and the accuracy of the closed forms is demonstrated using simulations. In Section 5, the sum rate results are used to derive a mode switching criterion. In Section 6, various results are shown to demonstrate the accuracy of the cooperation region obtained from derivations and the effect of different parameters on the region. The paper is concluded in Section 7.

\subsection{Notation}

In this paper, $(\cdot)^{H}$ and $\|\cdot\|$ refer to the conjugate transpose and norm, respectively. $\mathcal{C N}(\mathbf{0}, \mathbf{I})$ refers to independent and identically distributed (i.i.d.) complex Gaussian distribution with 0 mean and unit variance. $\mathbb{E}\{\cdot\}$ refers to expectation over the distribution of the channel, unless specified otherwise. The abbreviations BS, BSs, and $\mathrm{BS}_{k}$ denote 'base station', 'base stations', and 'the $k^{\text {th }}$ base station', respectively. 


\section{System model}

Consider a downlink cellular MISO system as shown in Figure 1 consisting of $K$ cooperating cells. Each cell contains a base station having $N_{t}$ transmit antennas and a single-antenna user. An orthogonal intra-cell multiple access scheme is assumed so that each BS serves only a single user per resource block. The channel between $\mathrm{BS}_{i}$ and user $k$ is denoted by a $1 \times N_{t}$ row vector $\mathbf{h}_{i k}$.

Each user estimates the direct channel from its serving BS ('local channel') and interfering channels from other BSs ('cross channels') using downlink pilot symbols. It is assumed that these channels can be estimated with negligible error at the users. Each user $k$ then feedbacks both its local channel $\left(\mathbf{h}_{k k}\right)$ and cross channels $\left(\mathbf{h}_{i k}, \forall i \neq k\right)$ to its serving base station, $\mathrm{BS}_{k}$, as shown in Figure 1. Then, the BSs exchange the cross channel information through the back-haul link. This feedback scheme is compliant to single-cell systems and the requirement that users communicate to their serving BSs only [16]. It is also similar to the feedback scheme used in the LTE framework [6].

In practice, there is a delay before the BSs acquire the necessary channel state information. First, there is a feedback delay until each BS receives CSI from the local user, which affects all channel vectors. Then, there is a backhaul delay while exchanging CSI through the back-haul, which affects only the cross-channels. Assuming that the back-haul delay between all BS pairs is equal, the delay $D_{i k}$ incurred by each channel vector $\mathbf{h}_{i k}$ is given by

$$
D_{i k}= \begin{cases}D_{f} & \text { if } i=k \\ D_{f}+D_{b} & \text { if } i \neq k\end{cases}
$$

where $D_{f}$ and $D_{b}$ denote the feedback and back-haul delays, respectively. Let us denote $D_{f}+D_{b}$ by $D_{f+b}$ for notational brevity.

\subsection{Modeling the effect of delay}

In order to analyze the impact of delay, a model for the temporal variation of the channel is used. Consider the channel vector $\mathbf{h}_{i k}[n]$ and its delayed version $\mathbf{h}_{i k}\left[n-D_{i k}\right]$ where $n$ denotes a symbol time index. Assuming isotropic scattering and reception, the entries of $\mathbf{h}_{i k}$ can be modeled as i.i.d. complex Gaussian random variables, $\mathbf{h}_{i k} \sim \mathcal{C N}(\mathbf{0}, \mathbf{I})$, and the temporal correlation $\rho$ between the entries of $\mathbf{h}_{i k}[n]$ and $\mathbf{h}_{i k}\left[n-D_{i k}\right]$ can be given by

$$
\rho_{i k}=J_{0}\left(2 \pi f_{d} \tau D_{i k}\right)
$$

according to Clarke's autocorrelation model [20], where $f_{d}$ is the Doppler spread, $\tau$ is the symbol time, and $J_{0}(\cdot)$ is the zeroth-order Bessel function of the first kind. The tempo-

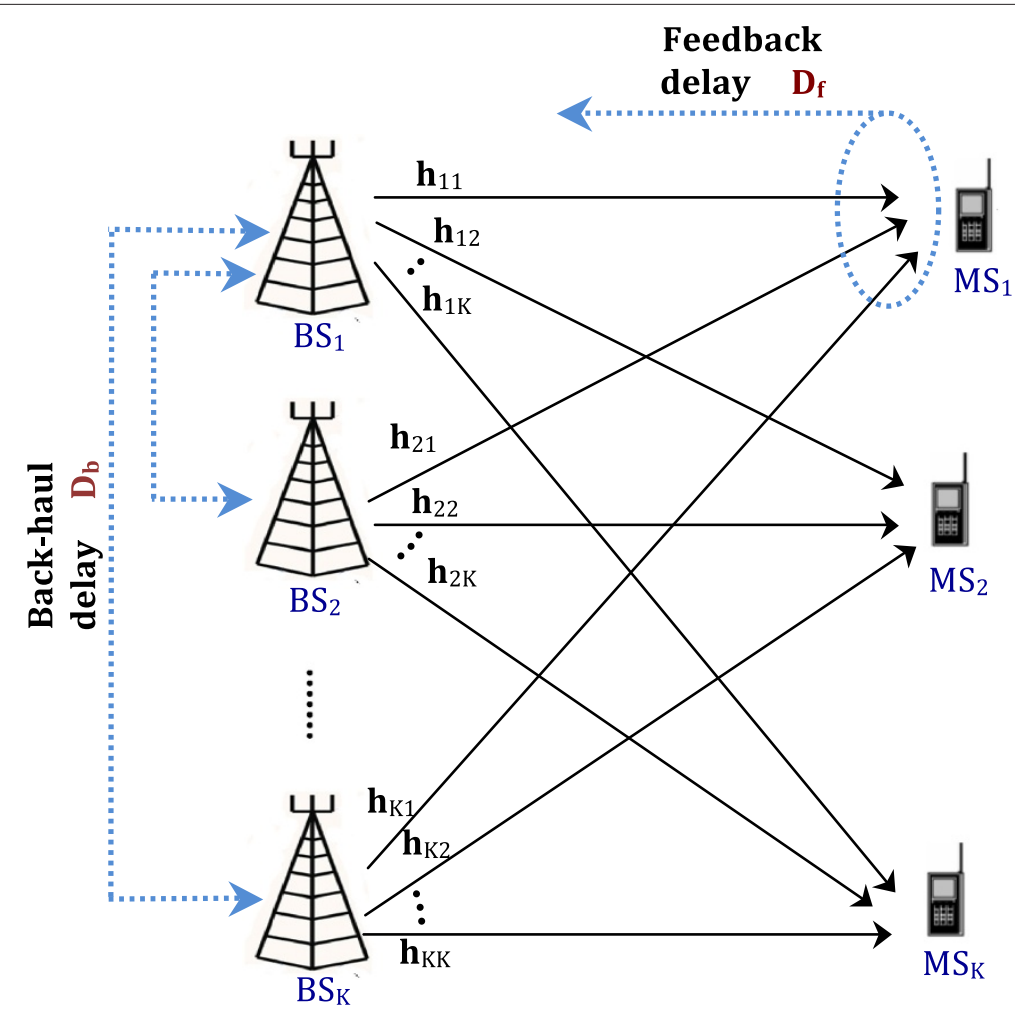

Figure 1 A downlink MISO-IC with $K$ cells each having an $N_{t}$ antenna base station. 
ral correlation is determined by the product $f_{d} \tau$, which is referred to as normalized Doppler frequency.

In slowly varying wireless channels, the Gauss-Markov autoregressive order one (AR1) model can represent the temporal variation with sufficient accuracy [21]. Using this model, $\mathbf{h}_{i k}[n]$ can be expressed as

$$
\mathbf{h}_{i k}[n]=\rho_{i k} \mathbf{h}_{i k}\left[n-D_{i k}\right]+\sqrt{1-\rho_{i k}^{2}} \mathbf{e}_{i k}[n]
$$

where $\mathbf{e}_{i k}[n] \sim \mathcal{C N}(\mathbf{0}, \mathbf{I})$ is a channel error vector independent of $\mathbf{h}_{i k}\left[n-D_{i k}\right]$.

\subsection{Limited feedback model}

Delay is not the only factor for channel state information inaccuracy. In practice, a limited feedback scheme with finite codebook size is used for quantizing the channel vectors in FDD systems [17]. Thus, the effect of quantization error caused by finite codebooks has to be considered. Even though the main goal of the paper is to study the effect of feedback delay and back-haul delay, the effect of limited feedback is also considered for completeness.

Random vector codebooks are commonly used for analyzing the effect of vector quantization schemes on the performance of MIMO systems [18,22]. Consider a random vector codebook of $B$-bits for quantizing each normalized channel direction $\tilde{\mathbf{h}}_{i k}:=\frac{\mathbf{h}_{i k}}{\left\|\mathbf{h}_{i k}\right\|}$. In such a random codebook of $B$ bits, the $2^{B}$ codeword vectors are isotopically distributed in an $N_{t}$ dimensional unit sphere. Let $\theta_{i k}$ be the angle between the normalized channel direction $\tilde{\mathbf{h}}_{i k}[n]$ and the quantized channel direction denoted by $\hat{\mathbf{h}}_{i k}[n]$. Then, $\tilde{\mathbf{h}}_{i k}[n]$ can be expressed in terms of $\hat{\mathbf{h}}_{i k}[n]$ as

$$
\tilde{\mathbf{h}}_{i k}[n]=\sqrt{1-Z_{i k}} \hat{\mathbf{h}}_{i k}[n]+\sqrt{Z_{i k}} \mathbf{s}_{i k}[n]
$$

where $\mathbf{s}_{i k}[n]$ is an isotropically distributed unit norm error vector orthogonal to $\hat{\mathbf{h}}_{i k}[n]$ and $Z_{i k}=\sin ^{2}\left(\theta_{i k}\right)$ is the quantization error magnitude and is independent of $\mathbf{s}_{i k}[n]$. In a codebook of size $B, Z_{i k}$ is the minimum of $2^{B}$ beta $\left(1, N_{t}-1\right)$ distributed random variables [18]. Incorporating the quantization error model in the GaussMarkov model in (2), the relation between the channel vector $\mathbf{h}_{i k}[n]$ and its quantized and delayed version $\hat{\mathbf{h}}_{i k}\left[n-D_{i k}\right]$ can be expressed as follows:

$$
\begin{aligned}
\mathbf{h}_{i k}[n]= & \rho_{i k}\left\|\mathbf{h}_{i k}\left[n-D_{i k}\right]\right\|\left(\sqrt{1-Z_{i k}} \hat{\mathbf{h}}_{i k}\left[n-D_{i k}\right]\right. \\
& \left.+\sqrt{Z_{i k}} \mathbf{s}_{i k}\left[n-D_{i k}\right]\right)+\sqrt{1-\rho_{i k}^{2}} \mathbf{e}_{i k}[n] .
\end{aligned}
$$

\subsection{Input-output model}

The Gauss-Markov model represents only the fast fluctuations of the channel assuming that the statistical param- eters of the channel vary so slowly that their fluctuation can be neglected. However, the relative magnitudes (channel gain ratios) of the local and cross channels as a result of difference in path loss cannot be neglected in a multicell scenario. These relative magnitudes are considered together with the transmit power as follows.

Assume that $\mathrm{BS}_{k}$ transmits symbol $s_{k}$ to its local user using a beamformer $\mathbf{w}_{k}$, which satisfies $\left\|\mathbf{w}_{k}\right\|=1$. Let the power transmitted from $\mathrm{BS}_{k}$ be denoted by $P_{k}$, which satisfies per base station power constraints. In addition, let the path loss from $\mathrm{BS}_{i}$ to user $k$ be $L_{i k}$. The signal received by user $k$ is then given by

$$
\begin{aligned}
y_{k}[n]= & \sqrt{\frac{P_{k}}{L_{k k}}} \mathbf{h}_{k k}[n] \mathbf{w}_{k}[n] s_{k}[n] \\
& +\sum_{i=1, i \neq k}^{K} \sqrt{\frac{P_{i}}{L_{i k}}} \mathbf{h}_{i k}[n] \mathbf{w}_{i}[n] s_{i}[n]+v_{k}[n]
\end{aligned}
$$

where $v_{k}[n]$ denotes the additive complex Gaussian noise of variance $\sigma^{2}$. The resulting instantaneous signalto-interference-and-noise ratio (SINR) of user $k$ and the ergodic sum rate $R$ can be respectively expressed as

$$
\begin{aligned}
\operatorname{SINR}_{k}[n] & =\frac{\left|\mathbf{h}_{k k}[n] \mathbf{w}_{k}[n]\right|^{2}}{\frac{1}{\gamma_{k}}+\sum_{i=1, i \neq k}^{K} \alpha_{i k}^{2}\left|\mathbf{h}_{i k}[n] \mathbf{w}_{i}[n]\right|^{2}} \\
R & =\sum_{k=1}^{K} \mathbb{E}\left\{\log \left(1+\operatorname{SINR}_{k}[n]\right)\right\}
\end{aligned}
$$

where $\gamma_{k}$ represents the SNR at user $k$ and $\alpha_{i k}^{2}$ represents the ratio of the interference power from $\mathrm{BS}_{i}$ and the useful signal power from $\mathrm{BS}_{k}$.

$$
\begin{aligned}
\gamma_{k} & =\frac{P_{k} / L_{k k}}{\sigma^{2}} \\
\alpha_{i k} & =\sqrt{\frac{P_{i}}{P_{k}} \cdot \frac{L_{k k}}{L_{i k}}}
\end{aligned}
$$

In the case that interference can be mitigated by transmitter pre-processing, power control is not desired and maximum throughput can be obtained by transmitting with full power.

Assume that all BSs transmit with equal power and this results in a cell-edge SNR denoted by $\gamma_{E}$. Let us also denote the distance from $\mathrm{BS}_{i}$ to user $k$ by $d_{i k}$. Then, $\gamma_{k}$ and $\alpha_{i k}^{2}$ can be expressed as

$$
\begin{gathered}
\gamma_{k}=\gamma_{E}\left(\frac{r_{\text {cell }}}{d_{k k}}\right)^{\mu}=\frac{\gamma_{E}}{\left(d_{k k, N}\right)^{\mu}} \\
\alpha_{i k}^{2}=\left(\frac{d_{k k}}{d_{i k}}\right)^{\mu}=\left(\frac{d_{k k, N}}{d_{i k, N}}\right)^{\mu}
\end{gathered}
$$


where $r_{\text {cell }}$ is the cell radius and $\mu$ is the path loss exponent. $d_{k k, N} \in(0: 1]$ and $d_{i k, N}$ are the normalized distances (normalized to the cell radius) of user $k$ from its serving $\mathrm{BS}$ and an interfering $\mathrm{BS}_{i}$, respectively. When user $k$ is at the cell edge $\left(d_{k k, N}=1\right)$, its SNR $\gamma_{k}$ and the celledge SNR $\gamma_{E}$ become equal. Since a user is normally assigned to the BS from which it receives the strongest signal, the ratio $\frac{d_{k k}}{d_{i k}}$ is less than 1 . Hence, it holds in general that $\alpha_{i k}=1$ for $i=k$ and $\alpha_{i k}<1$ for $i \neq k$. Thus, the value of $\alpha_{i k}$ referred hereafter refers to the case of $i \neq k$ only.

\section{Competition versus cooperation}

In MISO-IC beamforming, there are two well-known categories of distributed beamforming strategies, namely competitive (egoistic) and cooperative (altruistic). Several beamforming schemes can be categorized under these strategies; however, the fundamental ones are maximum ratio transmission and inter-cell interference cancellation [11]:

- Maximum ratio transmission (MRT): In MRT, each BS ignores the inter-cell interference it causes to other cells and aims to maximize the received signal power at its intra-cell user. In MRT, the beamforming vector at $\mathrm{BS}_{k}$ with perfect CSI is given by

$$
\mathbf{w}_{k, e g}[n]=\frac{\mathbf{h}_{k k}^{H}[n]}{\left\|\mathbf{h}_{k k}[n]\right\|}
$$

where the subscript eg refers to the beamformer being egoistic. In MRT, the only CSI in need is the local channel; hence, there is less feedback overhead.

- Inter-cell interference cancellation (ICIC): In ICIC, the focus of each BS is to avoid interference caused to other cells. In the presence of enough transmit antennas $\left(N_{t} \geq K\right)$, the inter-cell interference can be completely nullified. As a result, the ICIC beamformer at $\mathrm{BS}_{k}$ can be expressed as

$$
\mathbf{w}_{k, a l}[n]=\frac{\boldsymbol{\Pi}_{k}^{\perp}[n] \mathbf{h}_{k k}^{H}[n]}{\left\|\boldsymbol{\Pi}_{k}^{\perp}[n] \mathbf{h}_{k k}^{H}[n]\right\|}
$$

where the subscript al denotes the beamformer being altruistic and $\Pi_{k}^{\perp}[n]$ is a projection matrix onto the subspace orthogonal to the channels being interfered. When $N_{t}<K$, projection in the direction of the smallest eigenvector of the interference subspace can be used instead.

MRT and ICIC are two extreme beamforming strategies. So, other beamforming schemes that optimize performance based on various criteria can be used to approach the capacity region of the MISO-IC. Some examples are virtual SINR maximization [23,24], MMSE estimation [25], and transmit power minimization [26]. The algorithms in [24-26] are iterative and require interBS information exchange. Most importantly, all these beamforming schemes require full channel knowledge. Specifically, both the magnitude and direction of the channel vectors are required for beamforming. Since only the normalized directions of the local and cross channels are fed back as discussed in Section 2.2, ICIC is the most suitable and practical cooperative beamforming scheme for such limited feedback systems. Similarly, having knowledge of only the directions of the local channels, MRT is the sum rate maximizing strategy for non-cooperative systems. Therefore, MRT and ICIC are chosen as the competitive and cooperative beamforming schemes in this paper. Therefore, we refer to MRT and ICIC, respectively, when we say egoistic beamforming (denoted by 'EgBf') and altruistic beamforming (denoted by 'AlBf') throughout this paper.

In the presence of full channel knowledge, it is known that ICIC achieves a higher sum rate than MRT at high SNR and MRT is better at low SNR [11]. However, it is not clear when and whether ICIC provides a sum rate improvement over MRT in the presence of quantized and delayed CSI. In the next section, closedform expressions for the average sum rates achieved by these beamformers are derived to study this basic problem.

\section{Sum rate analysis with delay and limited feedback}

Consider the feedback scheme shown in Figure 1. In order to use EgBf, only the local channels are used. On the other hand, in order to cooperate and use AlBf, both the local and cross channels are involved as shown in (6). Each BS receives an updated version of its local channel after a delay $D_{f}$, while all the cross channels have a delay of $D_{f+b}$. Thus, the channel correlation $\rho_{i k}$ in (3) can be replaced by the local channel correlation $\rho_{l}$ for $i=k$ and the cross channel correlation $\rho_{c}$ for $i \neq k$ where

$$
\begin{aligned}
& \rho_{l}=J_{0}\left(2 \pi f_{d} \tau D_{f}\right) \\
& \rho_{c}=J_{0}\left(2 \pi f_{d} \tau D_{f+b}\right) .
\end{aligned}
$$

Let us also consider the effect of limited feedback on these beamforming schemes. Assume that the total number of bits available for CSI feedback is denoted by $B$ and is the same at all users. For $\mathrm{EgBf}$, each user can use all the $B$-bits to quantize its local channel. When AlBf is used, on the other hand, the $B$-bits must be allocated between the local and cross channels. Various methods of quantization such as joint quantization [27] and separate quantization 
with adaptive bit allocation [16] may be considered for quantizing the local and cross channels. In this paper, separate quantization with equal bit allocation is used due to its simplicity and since the performance gained by adaptive bit allocation is only marginal [19]. Therefore, the number of bits used for quantizing each channel vector is equal to $\frac{B}{K}$ when AlBf is used. Though the same codebook size can be used, codebooks must be different from cell to cell to avoid the possibility of the same codebook vector selection.

Using the channel model in (3), the sum rate achieved with quantized and delayed CSI can be evaluated. The average sum rates achieved by EgBf and AlBf, denoted $R_{e g}$ and $R_{a l}$, respectively, can be expressed as follows:

$$
\begin{aligned}
& R_{e g}=\mathbb{E}\left\{\sum_{k=1}^{K} \log \left(1+\frac{\left|\mathbf{h}_{k k}[n] \mathbf{w}_{k, e g}[n]\right|^{2}}{\frac{1}{\gamma_{k}}+\sum_{i=1, i \neq k}^{K} \alpha_{i k}^{2}\left|\mathbf{h}_{i k}[n] \mathbf{w}_{i, e g}[n]\right|^{2}}\right)\right\} \\
& R_{a l}=\mathbb{E}\left\{\sum_{k=1}^{K} \log \left(1+\frac{\left|\mathbf{h}_{k k}[n] \mathbf{w}_{k, a l}[n]\right|^{2}}{\frac{1}{\gamma_{k}}+\sum_{i=1, i \neq k}^{K} \alpha_{i k}^{2}\left|\mathbf{h}_{i k}[n] \mathbf{w}_{i, a l}[n]\right|^{2}}\right)\right\}
\end{aligned}
$$

where the beamformers are given by ${ }^{\mathrm{a}}$ :

$$
\begin{aligned}
\mathbf{w}_{k, e g}[n] & =\hat{\mathbf{h}}_{k k}^{H}\left[n-D_{f}\right] \\
\mathbf{w}_{k, a l}[n] & =\frac{\Pi_{k}^{\perp}\left[n-D_{f+b}\right] \hat{\mathbf{h}}_{k k}^{H}\left[n-D_{f}\right]}{\left\|\boldsymbol{\Pi}_{k}^{\perp}\left[n-D_{f+b}\right] \hat{\mathbf{h}}_{k k}^{H}\left[n-D_{f}\right]\right\|} .
\end{aligned}
$$

In general, it is difficult to find an accurate and generalized closed-form expression for these ergodic sum rates. The main goal of this paper however is to determine which beamforming scheme (EgBf or AlBf) achieves a higher sum rate for a particular set of system and channel parameters such as SNR, Doppler frequency, delay, and codebook size. Therefore, two different sum rate representations, a lower bound and a first order approximation, are used to estimate and compare the sum rates.

\subsection{Sum rate representation using lower bound}

One possibility to get insight into and compare the ergodic sum rates is using a lower bound. In order to derive the lower bound, the concavity of the logarithmic function is used and high channel correlations are assumed. In a low-mobility environment, where the channels are slowly time-varying, this assumption is valid. The lower bound is stated in the following theorem.
Theorem 1. The ergodic sum rates in egoistic and altruistic beamforming can be lower bounded by the following closed-form expressions in the low-Doppler frequency region.

$$
\begin{aligned}
R_{e g}> & \frac{K}{\ln (2)}\left\{\psi\left(N_{t}\right)+\ln \left(\rho_{l}^{2}\right)-Q\right\} \\
& +\sum_{k=1}^{K} \log \left(\gamma_{k}\right)-\sum_{k=1}^{K} \log \left(1+\beta_{k}\right) \\
R_{a l}> & \frac{K}{\ln (2)}\left\{\psi\left(N_{t}-K+1\right)+\ln \left(\rho_{l}^{2}\right)-Q^{\frac{1}{K}}\right\} \\
& +\sum_{k=1}^{K} \log \left(\gamma_{k}\right)-\sum_{k=1}^{K} \log \\
& \times\left(1+\left(1-\rho_{c}^{2}+\rho_{c}^{2} \frac{N_{t}}{N_{t}-1} Q^{\frac{1}{K}}\right) \beta_{k}\right)
\end{aligned}
$$

where $B, \rho_{l}$, and $\rho_{c}$ are the codebook size, local channel correlation and cross-channel correlation, respectively, and $Q=2^{-\frac{B}{N_{t}-1}} \cdot \psi(x)$ is the digamma function given by $\psi(x)=\frac{d}{d x} \ln \Gamma(x)$, and $\beta_{k}$ is the total interference to noise ratio (INR) at user k given by

$$
\begin{aligned}
\beta_{k} & =\gamma_{k} \sum_{i=1, i \neq k}^{K} \alpha_{i k}^{2} \\
& =\gamma_{E} \sum_{i=1, i \neq k}^{K} \frac{1}{d_{i k, N}^{\mu}} .
\end{aligned}
$$

The proof is shown in Appendix 1. The distance of a user from an interfering base station is at least equal to the cell radius; thus, $d_{i k, N} \geq 1, \forall i \neq k$. If only neighboring BSs are interfering, then the maximum value of $d_{i k, N}$ is also 2 . Therefore, the value of $\beta_{k}$ can be bounded by $\gamma_{E}(K-1)(0.5)^{\mu}<\beta_{k} \leq \gamma_{E}(K-1)$.

The lower bound in Theorem 1 has good accuracy for the purpose of comparing the performance of EgBF and AlBF as will be shown in Section 6. However, it is too loose to estimate the actual value of the ergodic sum rates. Moreover, the assumption of slowly time-varying channels used in the derivation of the lower bound limits its generality. Therefore, a sum rate representation that can be used to estimate the ergodic sum rates in all parameter ranges is needed.

\subsection{Sum rate representation using first-order approximation}

A general representation of the ergodic sum rates in EgBf and AlBf can be obtained using approximations of the logarithmic function. Using first-order approximation of the logarithm of ratios, the approximate ergodic sum rates can be obtained as stated in the following theorem. 
Theorem 2. The first-order approximation of the ergodic sum rate achieved in egoistic and altruistic beamforming schemes with a B-bit codebook per user, a local channel correlation $\rho_{l}$ and cross channel correlation $\rho_{c}$ is given by

$$
\begin{aligned}
& R_{e g} \approx \sum_{k=1}^{K} \log \left\{1+\overline{\operatorname{SINR}}_{e g}\right\} \\
& R_{a l} \approx \sum_{k=1}^{K} \log \left\{1+\overline{\operatorname{SINR}}_{a l}\right\}
\end{aligned}
$$

where

$$
\begin{aligned}
& \overline{\operatorname{SINR}}_{e g}=\frac{\rho_{l}^{2}\left(N_{t}-1-N_{t} Q\right)+1}{\frac{1}{\gamma_{k}}\left(1+\beta_{k}\right)} \\
& \overline{\operatorname{SINR}}_{a l}=\frac{\rho_{l}^{2}\left(N_{t}-K-Q^{\frac{1}{K}}\left(N_{t}-K+1-\frac{K-1}{N_{t}-1}\right)\right)+1}{\frac{1}{\gamma_{k}}\left(1+\beta_{k}\left(1-\rho_{c}^{2}+\rho_{c}^{2} \frac{N_{t}}{N_{t}-1} Q^{\frac{1}{K}}\right)\right)} .
\end{aligned}
$$

The proof of this theorem is shown in Appendix 2.

Let us discuss two important points based on Theorems 1 and 2 .

1. The average interference power in EgBf is independent of the the number of quantization bits $B$ and the channel correlation. This is logical since the quality of CSI in other cells has no impact on in-cell performance if there is no cooperation.

2. In perfect CSI $\left(\rho_{c} \rightarrow 1, B \rightarrow \infty\right)$, the average interference power in AlBf vanishes since the beamforming direction is orthogonal to the interference subspace. However, that will not be the case if $N_{t}<K$. In the case of $N_{t}<K$, the average signal power remains the same as the case of $N_{t}=K$, whereas there will be a residual (additional) interference proportional to the smallest eigenvalue of the interference subspace. As a result, the average sum rate for AlBf will be lower than the one in Theorem 2.

\subsection{Simulation results: sum rate lower bound and approximation}

In order to validate the accuracy and suitability of the lower bound and approximation for representing the sum rate, a three-cell system as shown in Figure 2 is considered. In this three-cell system, the hexagonal area shown is cooperatively served. Such an area is typical of cooperation using sectored BS antennas in which each sector antenna serves one third of a cell. Such a three-cell system with sector antenna is commonly used in the literature $[19,24]$. We assume each BS to have four transmit antennas.
In practical scenarios, the location of each user is random, but uniformly distributed, within each cell. Therefore, it is assumed that the location of each of users 1,2 and 3 in Figure 2 is random and uniformly distributed within the sectors in their corresponding cells. The numerical results in this paper are obtained by averaging over large sets of such random users' locations; specifically, 100 tuples of random locations are averaged in most of the results. A path loss exponent of 3.7 is also used throughout the paper; this value is typical in practical channel models $[16,19]$.

In Figures 3 and 4, the sum rates of both EgBf and AlBf are shown vs. Doppler frequency and vs. SNR, respectively. In both figures, the sum rates obtained from Monte Carlo simulations are compared with the lower bound in Theorem 1 and the first-order approximation in Theorem 2 . The sum rates are averaged over the random locations of users 1, 2 and 3. It is assumed that the feedback delay and back-haul delay are each 1 symbol time; the quantization error is ignored. Another sum rate comparison is also shown in Figure 5 where the variation of the sum rate with distance is demonstrated. In this comparison, it is assumed that user 3 is located on the line connecting $\mathrm{BS}_{3}$ with the intersection point of the three cells in Figure 2, while the locations of users 1 and 2 are still uniformly distributed in their corresponding cells. Thus, the effect of the distance of user 3 from the cell center is demonstrated in Figure 5 by averaging the sum rates over the random locations of users 1 and 2 . The following points can be observed from Figures 3, 4 and 5:

1. The first-order approximation has a good accuracy in estimating the true sum rates for both EgBf and AlBf. However, it can be observed that the approximation is tighter for EgBf than for AlBf. This becomes a limitation in deciding the mode switching points. In fact, it can be observed that the crossing point of the lower bounds is closer to (is thus more accurate in estimating) the crossing point of the true sum rates than that of the first-order approximations.

2. The impact of CSI inaccuracy, in this case delay, is much severe in AlBf than EgBf. In general, cooperation yields a lower throughput than competition at higher Doppler frequency and low SNR, confirming that cooperation is not always helpful.

3. It can be observed from Figure 5 that an increase in distance from the cell center has similar impact on both beamforming schemes. This is based on the assumption that SNR decreases as a user moves towards a cell edge (away from its serving BS) as shown in (4). If the same SNR can be received at all locations, it will be EgBf that will be severely affected as a user moves towards the cell edge. 


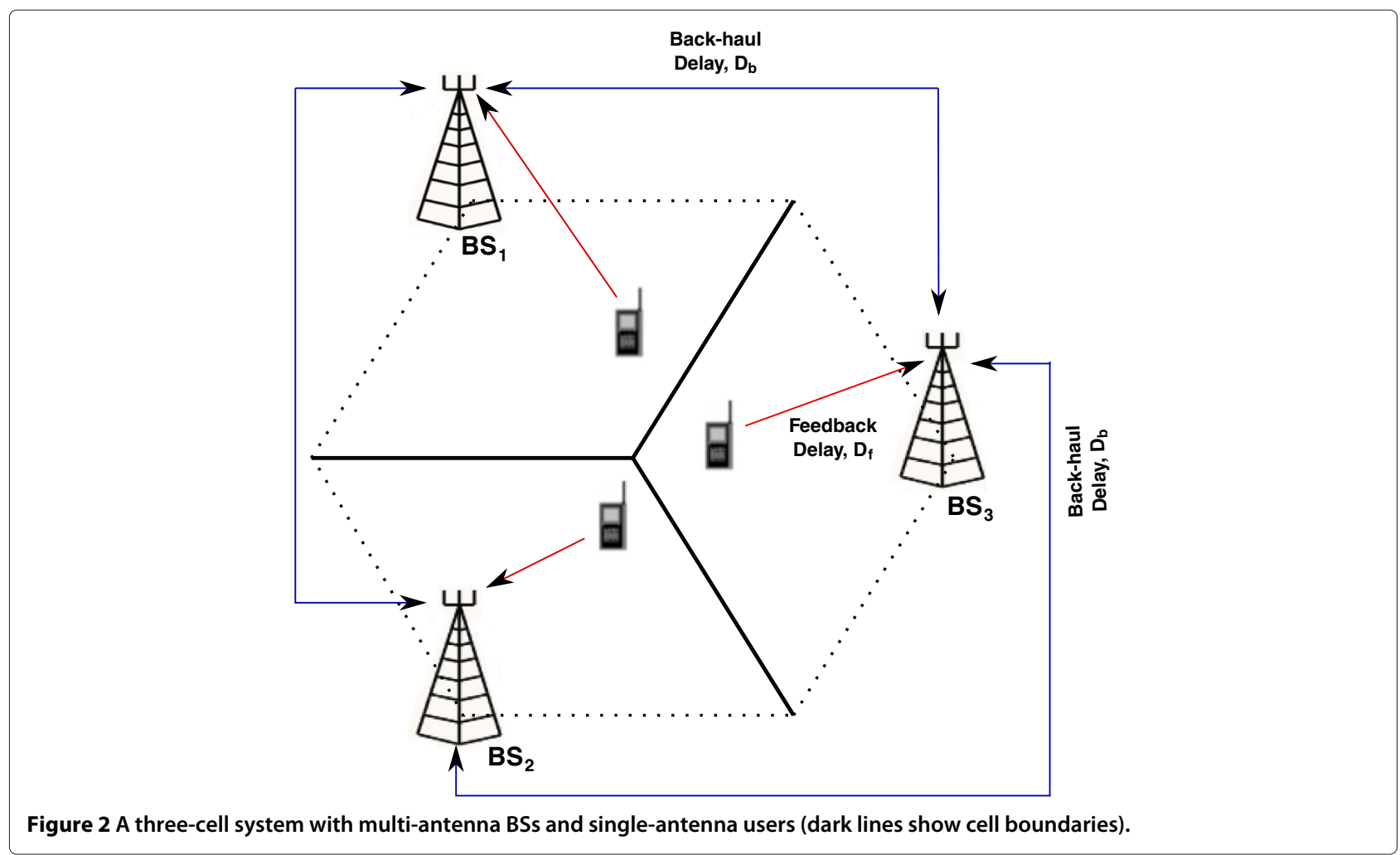

\section{Mode switching with delay and codebook size}

One of the goals of this paper is to obtain a decision criterion that allows a cluster of cells to switch between cooperation and competition in order to maximize performance for a given set of system parameters and feedback exchange properties. Various metrics can be considered for such a decision; however, sum rate is an appropriate

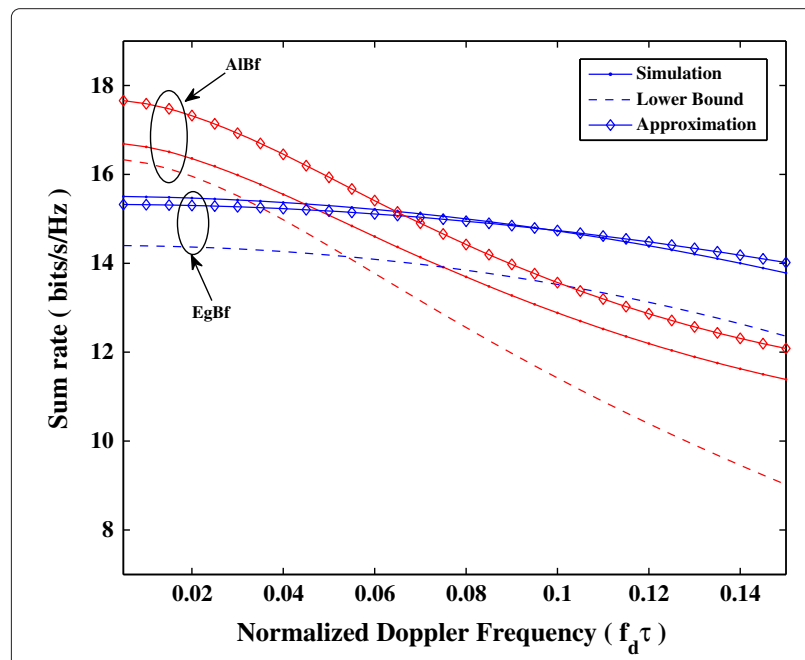

Figure 3 Average sum rate vs. Doppler frequency. With a cell edge SNR of $5 \mathrm{~dB}$, a feedback delay of 1 and a back-haul delay of 1 . metric since it encourages fairness besides maximizing throughput. When sum rate is used as a metric, a BS is forced to accept a scheme that benefits the whole cluster even though its local user can benefit from the opposite scheme. Consider the three-cell system in Figure 2 for instance; assume that user 1 is closer to the cell center while user 2 and user 3 are closer to the cell edge. If

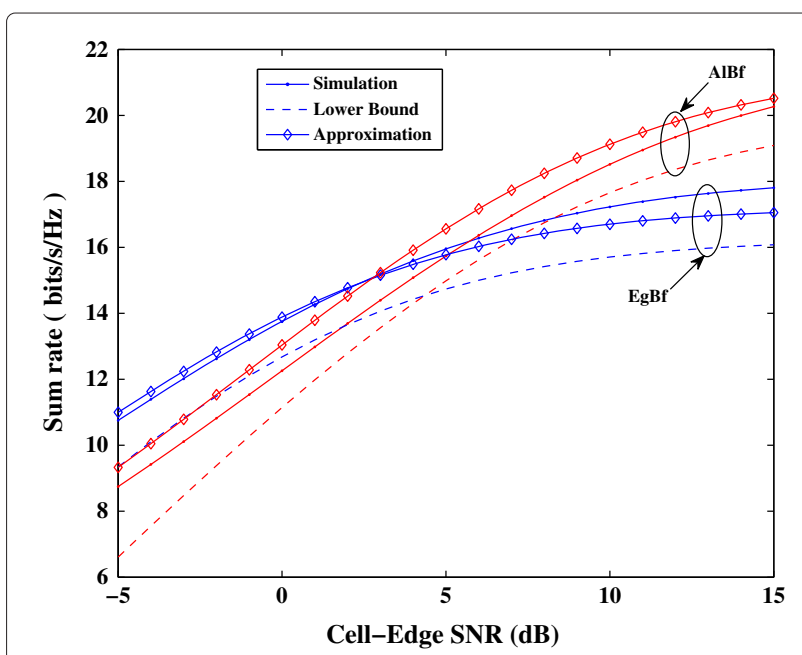

Figure 4 Average sum rate vs. cell edge SNR. With a Doppler frequency of 0.05, a feedback delay of 1 and a back-haul delay of 1 . 


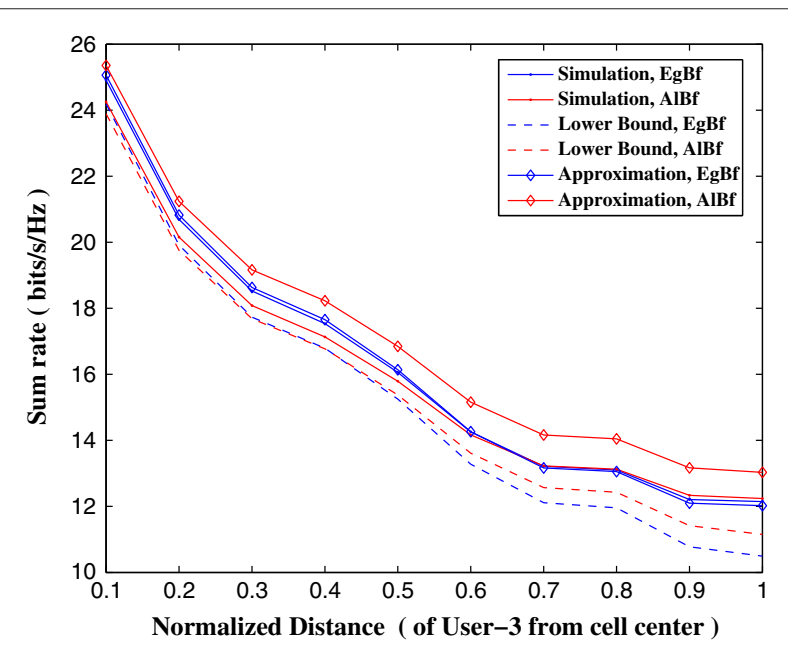

Figure 5 Average sum rate vs. normalized distance. With a cell edge SNR of $5 \mathrm{~dB}$, a Doppler frequency of 0.05 , a feedback delay of 1 and a back-haul delay of 1 .

decisions were to be made based on individual rate, $\mathrm{BS}_{1}$ would like to serve user 1 using EgBf even though this will cause significant interference to users 2 and 3. On the other hand, when sum rate is used as a metric, a beamforming scheme that maximizes the system throughput is chosen and that will probably be a fair scheme to users 2 and 3. Therefore, sum rate is selected as a metric and the parameter region where cooperative beamforming achieves a higher sum rate than competitive beamforming is discussed in this paper. This region is referred to as 'cooperation region' hereafter.

The cooperation region can be obtained by using the inequality $R_{e g}<R_{a l}$ for the actual sum rates. However, the cooperation region must be expressed in closed form as a function of the system and channel parameters so that the BSs can determine the operating mode based on these parameters. Therefore, the cooperation region is rather determined by using the corresponding lower bounds from Theorem 1 or first-order approximations from Theorem 2 instead of $R_{e g}$ and $R_{a l}$. Once a closedform expression characterizing the cooperation region is obtained, the operation mode can be switched between AlBf and EgBf depending on whether the cooperation region inequality is satisfied.

\subsection{Cooperation region}

The closed-form expressions from the lower bound are simpler to analyze and compare. Moreover, it is demonstrated in Section 4.3 that the lower bound has good accuracy in estimating the crossing point of the actual sum rates. Therefore, it is used here to study the the effect of various system and channel parameters on the cooperation region.
Using the lower bound in Theorem 1, the region where cooperation achieves a higher sum rate than competition $\left(R_{e g}<R_{a l}\right)$ is found to be ${ }^{\mathrm{b}}$

$$
\begin{aligned}
\sum_{k=1}^{K-1} & \frac{1}{N_{t}-k}<\left(Q-Q^{\frac{1}{K}}\right)+ \\
& \frac{1}{K} \sum_{k=1}^{K} \ln \left(\frac{1+\beta_{k}}{1+\left(1-\rho_{c}^{2}+\rho_{c}^{2} \frac{N_{t}}{N_{t}-1} Q^{\frac{1}{K}}\right) \beta_{k}}\right)
\end{aligned}
$$

where $\beta_{k}=\gamma_{E}\left(\sum_{i=1, i \neq k}^{K} \frac{1}{d_{i k, F}^{\mu}}\right)$. From this relation, the following points can be observed:

- In the low-SNR region $\left(\gamma_{E} \rightarrow 0\right), \sum_{k=1}^{K-1} \frac{1}{N_{t}-k}<Q-Q^{\frac{1}{K}}$ is obtained. This relation is always false since the right-hand side is negative and the left is positive. This shows that competition (EgBf) is preferred at low SNR irrespective of the quality of channel state information. This is expected since the system is noise-limited and performance can be enhanced only using MRT.

- On the other hand, in the high-SNR region $\left(\gamma_{E} \rightarrow \infty\right)$, $\sum_{k=1}^{K-1} \frac{1}{N_{t}-k}<Q-Q^{\frac{1}{K}}-\ln \left(1-\rho_{c}^{2}+\rho_{c}^{2} \frac{N_{t}}{N_{t}-1} Q^{\frac{1}{K}}\right)$ is obtained. Therefore, the choice of a beamforming scheme at high SNR depends on the quality of channel state information. In the case of high CSI quality $\left(\rho_{c} \rightarrow 1, B \rightarrow \infty\right)$, this reduces to $\sum_{k=1}^{K-1} \frac{1}{N_{t}-k}<\infty$ which confirms that cooperation is indeed beneficial at high SNR with good CSI quality. In this case, the system is interference-limited and performance can be enhanced by using ICIC.

- When the feedback delay, back-haul delay or Doppler frequency increases resulting in a lower $\rho_{c}$ in (12), the cooperation region shrinks. This is because AlBf is very sensitive to delay than EgBf.

- It is discussed that $\beta_{k}$ can be bounded by $\gamma_{E}(K-1)$ $0.5^{\mu}<\beta_{k} \leq \gamma_{E}(K-1)$. Therefore, $\beta_{k}$ does not significantly vary with distance. Hence, the cooperation region has less sensitivity to the location of users.

The cooperation region for a delay-free CSI feedback can be obtained using $\rho_{c}=1$ in (12). Similarly, the cooperation region in the case of high-quality quantization can be obtained by using $Q=0$ in (12).

The cooperation region in (12) depends on various system and channel parameters, namely the number of cooperating cells (cluster size), number of transmit antennas, SNR, codebook size, total delay, and Doppler frequency. Given knowledge of these parameters, the BSs can decide whether the cluster has to cooperate or compete 
in a decentralized, but synchronized way. Most of these parameters do not change with time. For parameters like SNR and Doppler frequency which may change slightly with time, a long-term (less frequent) feedback can be used to update them.

\section{Numerical results}

In this section, various numerical results on the multi-cell cooperation region are shown. In particular, the boundary of the cooperation region obtained from Monte Carlo simulations is compared with the boundaries obtained from closed-form expressions of the sum rate lower bound and approximation. The numerical results in this section consider two objectives. The first objective is to demonstrate the validity and accuracy of the lower bounds and first-order approximations for the purpose of mode switching (estimating the cooperation region boundary). The second objective is to investigate and verify the effect of various system and channel parameters on the cooperation region.

In Figures 6, 7, 8, 9 and 10, the effect of feedback delay and back-haul delay is considered; the quantization error is assumed to be negligible. Such results with CSI imperfection due to delay only help to understand the performance of FDD systems with large codebook sizes and TDD systems where limited feedback may not be necessary. Nonetheless, the effect of both delay and quantization is also demonstrated in Figure 11.

For all the numerical results, the three-cell system shown in Figure 2 is used. Each BS is assumed to have four transmit antennas except the result in Figure 9, in which the effect of the number of BS antennas is considered. The locations of the users are assumed to be random and

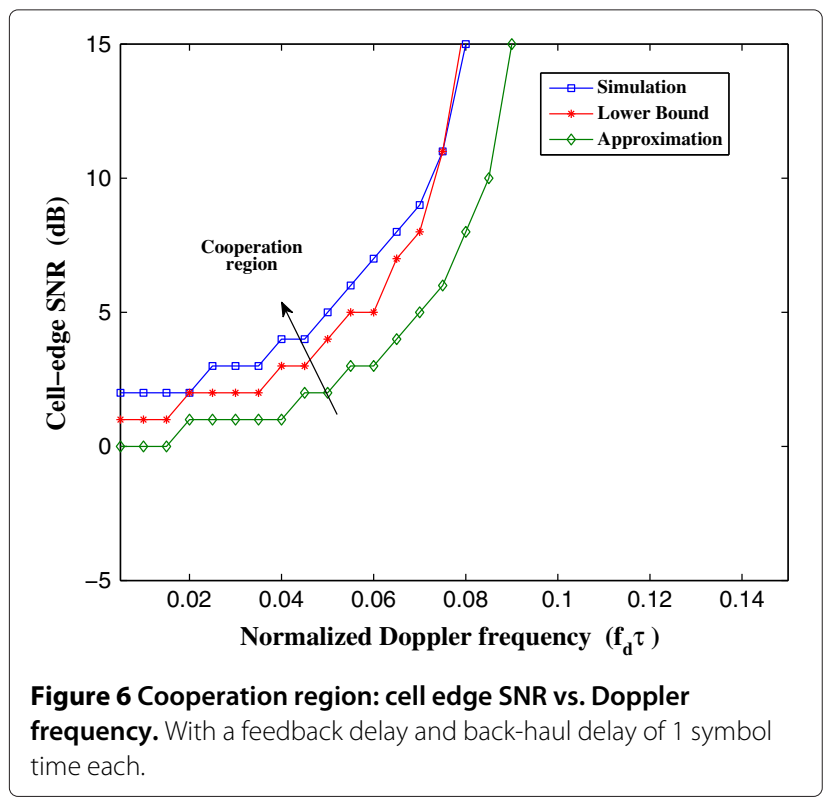

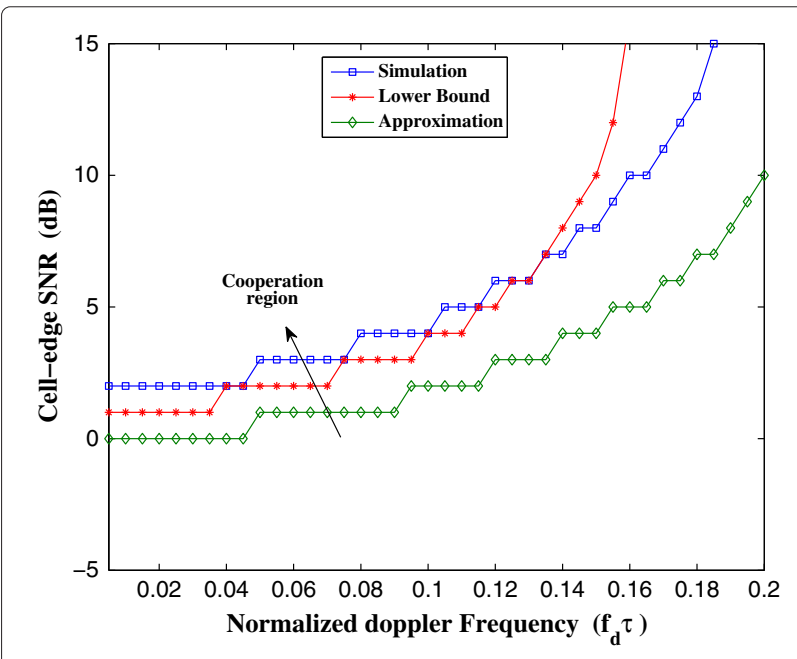

Figure 7 Cooperation region: cell edge SNR vs. Doppler frequency. With only a feedback delay of 1 symbol time.

uniformly distributed in each sector and a path loss exponent of 3.7 is used. All the numerical results are obtained by averaging over the random locations of all the users. The only exception is Figure 8, in which the effect of the distance of the third user from the cell center is demonstrated by averaging over the random locations of users 1 and 2 only (the same scenario as Figure 5).

\subsection{Validity of lower bound and first-order approximation of sum rates for mode switching}

In Figures 6 and 7, the validity of the lower bound and sum rate approximations for estimating the cooperation region boundary (mode switching points) is demonstrated

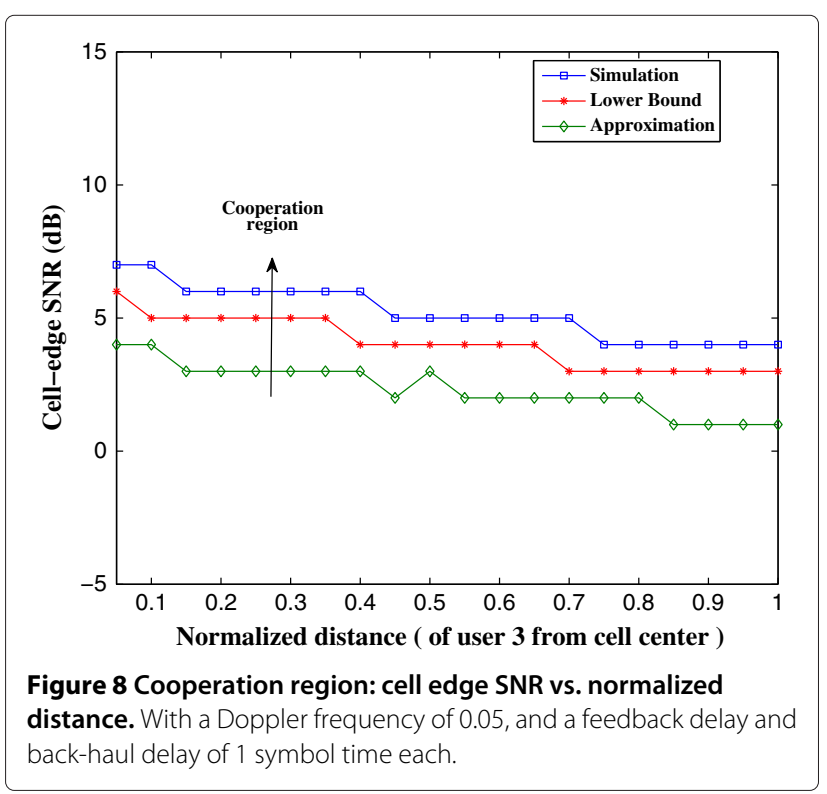




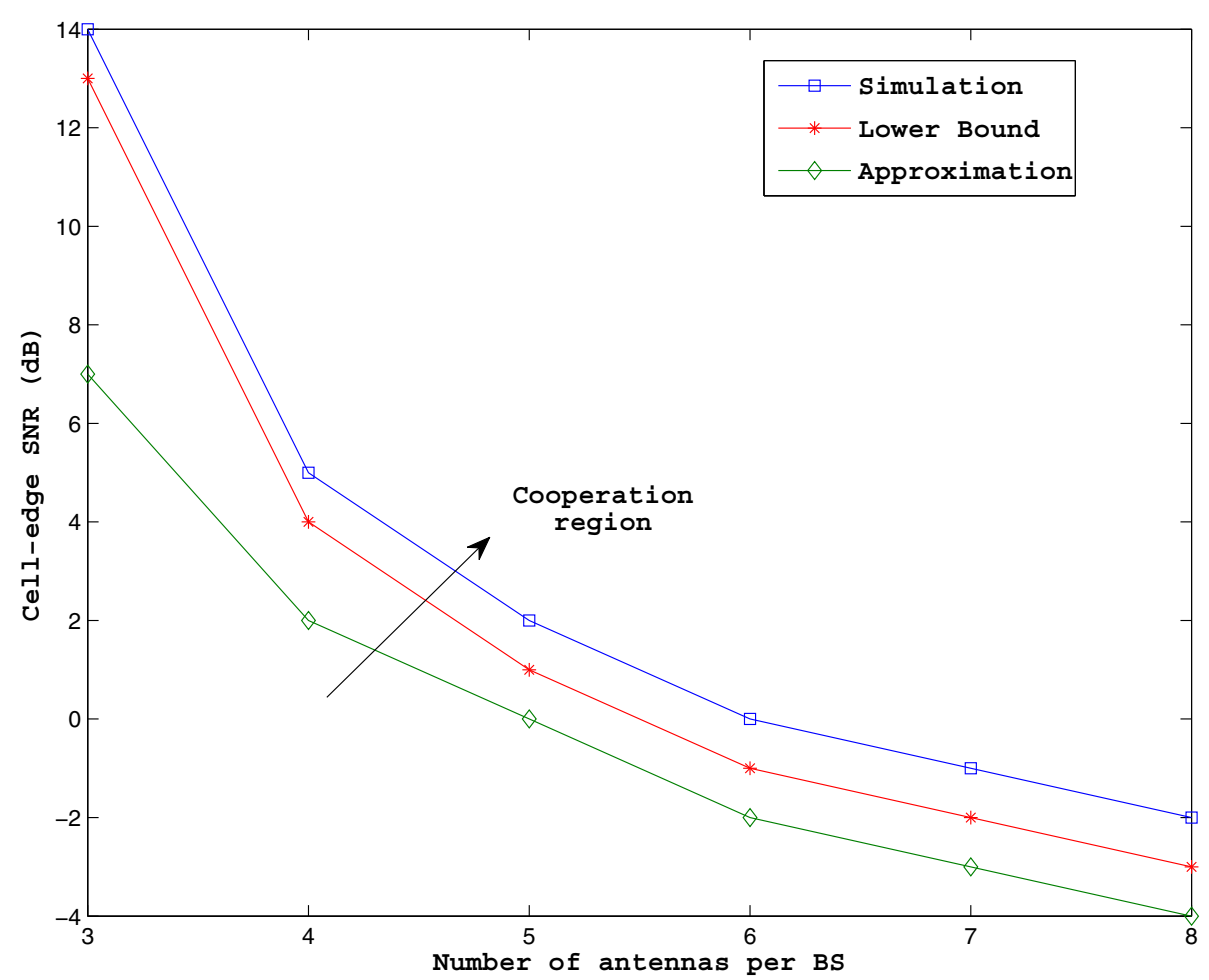

Figure 9 Cooperation region: cell edge SNR vs. number of transmit antennas. With a Doppler frequency of 0.05, and a feedback delay and back-haul delay of 1 symbol time each.

in the presence of feedback delay and back-haul delay. The cell-edge SNR vs. Doppler frequency cooperation region obtained from Monte Carlo simulations is compared with the region obtained from sum rate lower bounds and approximations in Theorem 1 and 2, respectively. In Figure 6, a feedback delay and back-haul delay of 1 symbol time each is assumed; on the other hand, only

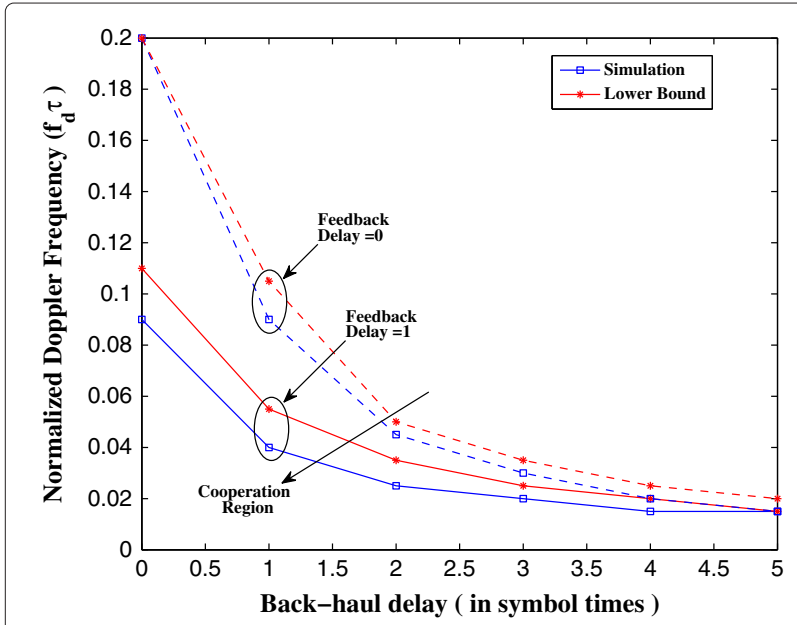

Figure 10 Cooperation region: Doppler frequency vs. back-haul delay. At a cell edge SNR of $5 \mathrm{~dB}$. feedback delay is considered in Figure 7. The region in the left top side pointed by the arrow is the cooperation region where AlBf outperforms EgBf. From the figures, it can be observed that both the lower bound and first-order approximations are close to the simulation (have a reasonable accuracy). However, the lower bound estimates the cooperation region much better than the first-order

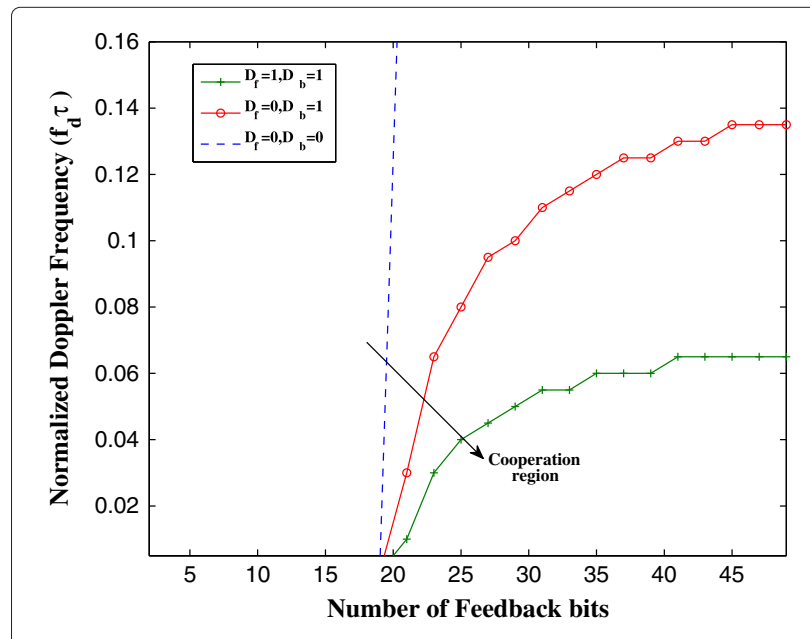

Figure 11 Cooperation region: Doppler frequency vs. number of feedback bits. At a cell edge SNR of $10 \mathrm{~dB}$. 
approximation in both figures. This can also be explained using Figures 3 and 4. While the first-order approximation has a good accuracy in estimating the sum rates in general, it slightly overestimates the sum rate in AlBf. This results in a significant error in determining the mode switching points. On the other hand, the error incurred by the lower bound has a similar pattern for both EgBf and AlBf; thus, the lower bound has better accuracy in determining the crossing points of the actual sum rates. However, the accuracy of the lower bound decreases with increase in Doppler frequency as shown in Figure 7 due to the high channel correlation assumption used in its derivation. Nevertheless, the lower bound has a good accuracy over typical normalized Doppler frequency ranges which rarely exceed 0.15 in practical multi-cell systems. The accuracy of the lower bound for estimating the cooperation region can also be confirmed from other comparisons in Figures 8 and 9.

\subsection{Effect of system and channel parameters on the cooperation region}

In Figures 6 and 7, the effect of cell-edge SNR and Doppler frequency on the cooperation region can be observed. In general, the cooperation region shrinks with increase in mobility (Doppler frequency). At low SNR, competition outperforms cooperation irrespective of the Doppler frequency. This is the same as the result obtained from the limit analysis in Section 5. On the other hand, the preferred beamforming mode at high SNR depends on the Doppler frequency, EgBf being preferred in high-mobility scenarios and AlBf in low mobility.

Another parameter that can have an effect on the cooperation region is the location of users. In Figure 8, the cooperation region is shown as a function of distance of user 3 from its corresponding base station; a Doppler frequency of 0.05 and a feedback and backhaul delay of 1 symbol time each are used. It can be observed that distance from the cell center has almost no impact on the cooperation region; the region is affected only by the cell-edge SNR, which is determined by the transmit power and the cell radius. The reason for the minimal impact of location of users can be explained as follows. When a user moves towards a cell edge (away from its local BS), the SNR it receives decreases due to path loss while the interference it encounters from other BSs increases. The decrease in SNR encourages the use of EgBf, while the increase in interference encourages the use of AlBf. Therefore, both the EgBf and AlBf sum rates suffer drastically as shown in Figure 5. Unless a constant SNR can be kept as a user moves towards the cell edge, there will be no clear winner between EgBf and AlBf.

Another system parameter that affects the cooperation region is the number of antennas per $\mathrm{BS}$ as compared to the number of users being served. This effect is demonstrated in Figure 9 where the number of transmit antennas at each BS is varied from 3 to 8 for the three-cell system in Figure 2. The feedback and back-haul delays are set to 1 symbol time each, and a Doppler frequency of 0.05 is assumed. As $N_{t}$ increases, the cooperation region expands owing to the fact that the system has more degrees of freedom to cancel the interference and at the same time maximize the received signal power. When the number of extra degrees of freedom (number of antennas minus number of cooperating cells, $N_{t}-K$ ) increases, the performance achieved with AlBf is boosted making it more preferable. On the other hand, if the number of antennas is less than the number of cooperating cells $\left(N_{t}<K\right)$, the region will shrink more due to a residual inter-cell interference which exists even with perfect channel state information.

The general effect of mobility and channel state information delay is shown in Figure 10 with a Doppler frequency vs. back-haul delay cooperation region. The values of the Doppler frequency, feedback delay, and backhaul delay together determine the local and cross channel correlations which affect the cooperation region. As expected, the figure shows that cooperation is preferred in low-mobility and low-delay scenarios and the cooperation region diminishes with increase in Doppler frequency or delay.

In all numerical simulations so far, the effect of quantization error is ignored. For completeness, the joint effect of quantization error and delay is demonstrated in Figure 11. For simplicity of demonstration, only the cooperation region obtained from lower bounds is plotted. Figure 11 shows the effect of increase in number of feedback bits (codebook size) on the cooperation region with different values of feedback delay $D_{f}$ and back-haul delay $D_{b}$. For small to medium codebook sizes, the figure shows that competitive beamforming is preferred irrespective of the Doppler frequency. This is based on the assumption the all feedback bits are used to represent only the local channel in EgBf while the same number of feedback bits are divided equally to represent the local and cross channels in AlBf. If the same codebook size can be used per channel vector in both schemes, the cooperation region will not be as pessimistically narrow as Figure 11. In that case, AlBf can outperform EgBf with codebook sizes in the order of 5 bits.

\section{Conclusions}

In this paper, the sum rate performance of two extreme competitive and cooperative beamforming schemes, namely MRT and ICIC, is evaluated and compared in the presence of quantized and delayed channel state information. Closed-form expressions are derived for a lower bound and a first-order approximation of the ergodic sum 
rates achieved by these beamforming strategies. Using the closed-form expressions, a mode switching criterion is proposed to switch between these beamforming strategies based on the SNR and quality of channel state information. It is shown that competitive beamforming is preferred at low SNR irrespective of the quality of channel state information. On the other hand, cooperative beamforming is preferred at high SNR only at low Doppler frequencies and large codebook sizes. This is because cooperative beamforming schemes require accurate information about the interfering channels to be able to nullify interference; however, such information is obtained after an extra back-haul delay resulting in further CSI inaccuracy. With small codebook sizes, competitive beamforming is preferred since it requires only a local channel information feedback which can be quantized with few bits. In addition, it is demonstrated that cooperative beamforming gains from the availability of more degrees of freedom. In general, it is shown that base station cooperation is not always advantageous.

In this paper, MRT and ICIC are selected as representative competitive and cooperative beamforming schemes. One of the motivations for such a choice is the suitability of these schemes for a limited feedback framework. Despite that, the performance analysis in this paper can be extended to other competitive and cooperative beamforming schemes. In addition, single-antenna users are considered in this paper. The sum rate analysis can be extended to multi-antenna users by considering suitable receive combining vectors. A re-consideration of these issues is left as a future work.

\section{Endnotes}

${ }^{a} \boldsymbol{\Pi}_{k}^{\perp}$ is used instead of $\hat{\boldsymbol{\Pi}}_{k}^{\perp}$ (the subspace orthogonal to quantized vectors) just for notational brevity.

${ }^{\mathrm{b}}$ The sequential property of the digamma function is used here to find a simplified expression.

${ }^{\mathrm{c}}$ All the cross products are of the form $\mathbf{a b}{ }^{H} \mathbf{b} \mathbf{c}^{H}$. For $t_{2} t_{3}^{*}$ and $t_{1} t_{3}^{*}$, all vectors are independent. For $t_{1} t_{2}^{*}$, a and $\mathbf{c}$ are orthogonal and independent. Hence, the average value of these products is zero since all vectors are i.i.d. zero-mean Gaussian.

\section{Appendices}

\section{Appendix 1: sum rate using lower bound}

A lower bound on the ergodic rates can be obtained using the following relation:

$$
\begin{aligned}
\mathbb{E}\left\{\log \left(1+\frac{x}{\frac{1}{\gamma}+y}\right)\right\} & \stackrel{a}{>} \mathbb{E}\{\log (x)\}-\mathbb{E}\left\{\log \left(\frac{1}{\gamma}+y\right)\right\} \\
& \stackrel{b}{>} \mathbb{E}\{\log (x)\}-\log \left(\frac{1}{\gamma}+\mathbb{E}\{y\}\right)
\end{aligned}
$$

where $x$ represents the signal power and $y$ represents the interference power. $(a)$ is due to the monotonicity of the logarithmic function and linearity of expectation and is tight in the high-SINR region. (b) is due to the concavity of the logarithmic function (Jensen's inequality). Using this relation, the lower bounds of the ergodic sum rates in (8) reduce to

$$
\begin{aligned}
R_{e g}> & \sum_{k=1}^{K} \mathbb{E}\left\{\log \left(\left|\mathbf{h}_{k k}[n] \mathbf{w}_{k, e g}[n]\right|^{2}\right)\right\}- \\
& \log \left(\frac{1}{\gamma_{k}}+\sum_{i=1, i \neq k}^{K} \alpha_{i k}^{2} \mathbb{E}\left\{\left|\mathbf{h}_{i k}[n] \mathbf{w}_{i, e g}[n]\right|^{2}\right\}\right) \\
R_{a l}> & \sum_{k=1}^{K} \mathbb{E}\left\{\log \left(\left|\mathbf{h}_{k k}[n] \mathbf{w}_{k, a l}[n]\right|^{2}\right)\right\}- \\
& \log \left(\frac{1}{\gamma_{k}}+\sum_{i=1, i \neq k}^{K} \alpha_{i k}^{2} \mathbb{E}\left\{\left|\mathbf{h}_{i k}[n] \mathbf{w}_{i, a l}[n]\right|^{2}\right\}\right) .
\end{aligned}
$$

\section{Egoistic beamforming}

In the case of EgBf, the CSI needed for beamforming experience only a feedback delay. Thus, both the local channels $\mathbf{h}_{k k}[n]$ and the cross channels $\mathbf{h}_{i k}[n]$ are modeled by using $\rho_{l}$ and $D_{f}$ in place of $\rho_{i k}$ and $D_{i k}$ in the Gauss-Markov model in (3).

For slowly time-varying channels or in cases where the feedback delay is small, the temporal correlation $\rho_{l}$ is close to 1 ; thus, it can be assumed that $\sqrt{1-\rho_{l}^{2}} \ll \rho_{l}$. Using this assumption, $\mathbf{h}_{k k}[n]$ can be approximated as

$$
\mathbf{h}_{k k}[n] \approx \rho_{l} \sqrt{1-Z_{k k}}\left\|\mathbf{h}_{k k}\left[n-D_{f}\right]\right\| \hat{\mathbf{h}}_{k k}\left[n-D_{f}\right] .
$$

The second term containing the vector $\mathbf{s}_{k k}\left[n-D_{f}\right]$ is ignored since it is orthogonal to $\mathbf{w}_{k, e g}[n]$ and does not have effect on $R_{e g}$. This approximation for $\mathbf{h}_{k k}[n]$ and the model for $\mathbf{h}_{i k}[n]$ are used together with the beamformers in (9) to evaluate each term in the ergodic sum rate.

\section{Signal power (EgBf)}

$$
\left|\mathbf{h}_{k k}[n] \mathbf{w}_{k, e g}[n]\right|^{2} \approx \rho_{l}^{2}\left(1-Z_{k k}\right)\left\|\mathbf{h}_{k k}\left[n-D_{f}\right]\right\|^{2}
$$

Applying the logarithm and expectation yields

$$
\begin{gathered}
\mathbb{E}\left\{\log \left(\left|\mathbf{h}_{k k}[n] \mathbf{w}_{k, e g}[n]\right|^{2}\right)\right\} \approx \log \left(\rho_{l}^{2}\right)+ \\
\mathbb{E}_{C}\left\{\log \left(1-Z_{k k}\right)\right\}+\mathbb{E}\left\{\log \left\|\mathbf{h}_{k k}\left[n-D_{f}\right]\right\|^{2}\right\} .
\end{gathered}
$$

The third term is a chi-square distributed random variable with degrees of freedom $2 N_{t}$. Thus, $\mathbb{E}\left\{\log \left(\left\|\mathbf{h}_{k k}\left[n-D_{f}\right]\right\|^{2}\right)\right\}=\frac{\psi\left(N_{t}\right)}{\ln (2)}$, where $\psi(x)$ is the digamma function given by $\frac{d}{d x} \ln (\Gamma(x))$. 
In $\mathbb{E}_{C}\left\{\log \left(1-Z_{k k}\right)\right\}$, the subscript $C$ denotes that the averaging is done on the set of random codebooks. It is shown in [18] that the expectation of the quantization error $Z_{k k}=\sin ^{2} \theta_{k k}$ is equal to $2^{B} \cdot \beta\left(2^{B}, \frac{N_{t}}{N_{t}-1}\right)$ in terms of the beta function, and it is shown to be upper bounded by $\mathbb{E}_{C}\left\{Z_{k k}\right\}<2^{-\frac{B}{N_{t}-1}}$ where $B$ is the size of the random codebook. For large codebook sizes, $Z_{k k}$ is small, so $\ln \left(1-Z_{k k}\right)$ can be approximated by $\ln \left(1-Z_{k k}\right) \approx-Z_{k k}$. Using this approximation and the upper bound of the quantization error, the average signal power is approximated as follows:

$$
\begin{aligned}
\mathbb{E}\left\{\log \left(\left|\mathbf{h}_{k k}[n] \mathbf{w}_{k, e g}[n]\right|^{2}\right)\right\} \\
\approx \log \left(\rho_{l}^{2}\right)-\frac{2^{-\frac{B}{N_{t}-1}}}{\ln (2)}+\frac{\psi\left(N_{t}\right)}{\ln (2)} .
\end{aligned}
$$

Interference power (EgBf) In order to evaluate the average interference power, the cross channel $\mathbf{h}_{i k}[n]$ is expressed in terms of $\hat{\mathbf{h}}_{i k}\left[n-D_{f}\right]$ and substituted in $\mathbb{E}\left\{\left|\mathbf{h}_{i k}[n] \mathbf{w}_{i, e g}[n]\right|^{2}\right\}$. Using the stationarity of the process to time-shift every vector by $D_{f}$, the following is obtained:

$$
\begin{aligned}
& \mathbb{E}\left\{\left|\mathbf{h}_{i k}[n] \mathbf{w}_{i, e g}[n]\right|^{2}\right\} \\
& =\mathbb{E}\left\{\left|\rho_{l} \sqrt{1-Z_{i k}} t_{0} t_{1}+\rho_{e g} \sqrt{Z_{i k}} t_{0} t_{2}+\sqrt{1-\rho_{l}^{2}} t_{3}\right|^{2}\right\} \\
& \stackrel{a}{=} \rho_{l}^{2}\left(1-\mathbb{E}_{C}\left\{Z_{i k}\right\}\right) \mathbb{E}\left\{t_{0}^{2}\right\} \mathbb{E}\left\{\left|t_{1}\right|^{2}\right\}+ \\
& \rho_{l}^{2} \mathbb{E}_{C}\left\{Z_{i k}\right\} \mathbb{E}\left\{t_{0}^{2}\right\} \mathbb{E}\left\{\left|t_{2}\right|^{2}\right\}+\left(1-\rho_{l}^{2}\right) \mathbb{E}\left\{\left|t_{3}\right|^{2}\right\}
\end{aligned}
$$

where $t_{0}:=\left\|\mathbf{h}_{i k}[n]\right\|, t_{1}:=\hat{\mathbf{h}}_{i k}[n] \hat{\mathbf{h}}_{i i}^{H}[n], t_{2}:=\mathbf{s}_{i k}[n] \hat{\mathbf{h}}_{i i}^{H}$ $[n]$, and $t_{3}:=\mathbf{e}_{i k}\left[n+D_{f}\right] \hat{\mathbf{h}}_{i i}^{H}[n]$. (a) is due to the fact that the norms of the channel vectors are independent of the quantization error $Z_{i k}$. In addition, all $t_{1}, t_{2}$, and $t_{3}$ are independent and the expectations of the cross products between them are 0 . The component terms have the following distribution: $\left|t_{1}\right|^{2}$ and $\left|t_{2}\right|^{2}$ are the squared projection of two independent vectors isotropically distributed on $N_{t}$-dimensional unit sphere; thus, $\left|t_{1}\right|^{2}$ and $\left|t_{2}\right|^{2}$ have beta distribution, Beta $\left(1, N_{t}-1\right)$, with mean $\frac{1}{N_{t}}[18] .\left|t_{3}\right|^{2}$ is the squared projection an isotropically distributed unit vector on an independent standard Gaussian vector; thus, $\left|t_{3}\right|^{2}$ has standard exponential distribution with mean 1 , as also stated in [11]. $t_{0}^{2}$ is chi-squared distributed with mean $N_{t}$.

Combining these expectations, the average interference power in EgBf reduces to

$$
\begin{aligned}
& \mathbb{E}\left\{\left|\mathbf{h}_{i k}[n] \mathbf{w}_{i, e g}[n]\right|^{2}\right\} \\
& =\rho_{l}^{2}\left(1-2^{-\frac{B}{N_{t}-1}}\right) N_{t} \cdot \frac{1}{N_{t}}+\rho_{l}^{2}\left(2^{-\frac{B}{N_{t}-1}}\right) N_{t} \cdot \frac{1}{N_{t}} \\
& \quad+\left(1-\rho_{l}^{2}\right) \\
& =1 .
\end{aligned}
$$

This shows that the interference caused to users in another cell is independent of the quality of channel state information in EgBf. This is expected since the beamformer is independent of the cross channels.

\section{Altruistic beamforming}

In the case of AlBf, both the local and cross channels are required. Since the local channels experience only a feedback delay, $\mathbf{h}_{k k}[n]$ is modeled as (13). On the other hand, the cross channel $\mathbf{h}_{i k}[n]$ is expressed in terms of $\hat{\mathbf{h}_{i k}}\left[n-D_{f+b}\right]$ by using $\rho_{c}$ and $D_{f+b}$ in (3).

\section{Signal power (AlBf)}

$$
\begin{aligned}
& \left|\mathbf{h}_{k k}[n] \mathbf{w}_{k, a l}[n]\right|^{2} \\
& =\left|\mathbf{h}_{k k}[n] \frac{\Pi_{k}^{\perp}\left[n-D_{f+b}\right] \hat{\mathbf{h}}_{k k}^{H}\left[n-D_{f}\right]}{\| \boldsymbol{\Pi}_{k}^{\perp}\left[n-D_{f+b}\right] \hat{\mathbf{h}}_{k k}^{H}\left[n-D_{f}\right] \mid}\right|^{2} \\
& \stackrel{a}{\approx} \rho_{l}^{2}\left(1-Z_{k k}\right)\left\|\mathbf{h}_{k k}\left[n-D_{f}\right]\right\|^{2}\left\|\boldsymbol{\Pi}_{k}^{\perp}\left[n-D_{f+b}\right] \hat{\mathbf{h}}_{k k}^{H}\left[n-D_{f}\right]\right\|^{2}
\end{aligned}
$$

(a) is obtained using the approximation for $\mathbf{h}_{k k}[n]$ and using the symmetry and squared property of projection matrices. Applying expectation and time shifting by $D_{f}$, the average signal power reduces to

$$
\begin{aligned}
& \mathbb{E}\left\{\log \left(\left|\mathbf{h}_{k k}[n] \mathbf{w}_{k, a l}[n]\right|^{2}\right)\right\} \\
& \approx \log \left(\rho_{l}^{2}\right)+\mathbb{E}_{C}\left\{\log \left(1-Z_{k k}\right)\right\}+ \\
& \mathbb{E}\left\{\log \left(\left\|\boldsymbol{\Pi}_{k}^{\perp}\left[n-D_{b}\right] \hat{\mathbf{h}}_{k k}^{H}[n]\right\| \mathbf{h}_{k k}[n]\|\|^{2}\right)\right\} .
\end{aligned}
$$

The re-constructed Gaussian vector $\left\|\mathbf{h}_{k k}[n]\right\| \hat{\mathbf{h}}_{k k}[n]$ has the same statistical properties as a standard Gaussian vector $\mathbf{h}_{k k}[n] .\left\|\boldsymbol{\Pi}_{k}^{\perp}\left[n-D_{b}\right] \mathbf{h}_{k k}^{H}[n]\right\|^{2}$ is the squared norm of projection of an $N_{t}$ dimensional vector $\mathbf{h}_{k k}[n]$ on a subspace of dimension $K-1$. Since $\Pi_{k}^{\perp}\left[n-D_{b}\right]$ is independent of $\mathbf{h}_{k k}[n]$, the squared norm of the projection is chi-square distributed with $2\left(N_{t}-K+1\right)$ degrees of freedom. Thus, $\mathbb{E}\left\{\log \left(\left\|\boldsymbol{\Pi}_{k}^{\perp}\left[n-D_{b}\right] \mathbf{h}_{k k}^{H}[n]\right\|^{2}\right)\right\}=$ $\frac{\psi\left(N_{t}-K+1\right)}{\ln (2)}$. In altruistic beamforming, each channel vector is assumed to be quantized with $\frac{B}{K}$ size codebook; hence, $\mathbb{E}_{C}\left\{\log \left(1-Z_{k k}\right)\right\} \approx 2^{-\frac{B}{K\left(N_{t}-1\right)}}$.

Combining these terms, the average signal power is evaluated as

$$
\begin{aligned}
& \mathbb{E}\left\{\log \left(\left|\mathbf{h}_{k k}[n] \mathbf{w}_{k, a l}[n]\right|^{2}\right)\right\} \\
& \approx \log \left(\rho_{l}^{2}\right)-\frac{2^{-\frac{B}{K\left(N_{t}-1\right)}}}{\ln (2)}+\frac{\psi\left(N_{t}-K+1\right)}{\ln (2)}
\end{aligned}
$$

Interference power (AlBf) Using the model for $\mathbf{h}_{i k}[n]$ expressed in terms of $\hat{\mathbf{h}_{i k}}\left[n-D_{f+b}\right]$ in $\mathbb{E}\left\{\mid \mathbf{h}_{i k}[n] \mathbf{w}_{i, a l}\right.$ $\left.\left.[n]\right|^{2}\right\}$, using the fact that $\hat{\mathbf{h}_{i k}}\left[n-D_{f+b}\right]$ is orthogonal to 
$\mathbf{w}_{i, a l}[n]$ and time shifting by $D_{f}$, the interference power can be expressed as

$$
\begin{aligned}
& \mathbb{E}\left\{\left|\mathbf{h}_{i k}[n] \mathbf{w}_{i, a l}[n]\right|^{2}\right\}= \\
& \rho_{c}^{2} \mathbb{E}_{C}\left\{Z_{i k}\right\} \mathbb{E}\left\{t_{0}^{2}\right\} \mathbb{E}\left\{\left|t_{2}\right|^{2}\right\}+\left(1-\rho_{c}^{2}\right) \mathbb{E}\left\{\left|t_{3}\right|^{2}\right\}
\end{aligned}
$$

where $t_{0}=\left\|\mathbf{h}_{i k}\left[n-D_{b}\right]\right\|, t_{2}=\mathbf{s}_{i k}\left[n-D_{b}\right] \frac{\Pi_{i}^{\perp}\left[n-D_{b}\right]_{i i}^{H}[n]}{\| \Pi_{i}^{\perp}\left[n-D b \mid \hat{\mathbf{h}}_{i i}^{H}[n] \|\right.}$ and $t_{3}=\mathbf{e}_{i k}\left[n+D_{f}\right] \frac{\Pi_{i}^{\perp}\left[n-D_{b}\right] \hat{\mathbf{h}}_{i i}^{H}[n]}{\left\|\Pi_{i}^{\perp}\left[n-D_{b}\right] \hat{\mathbf{h}}_{i i}^{H}[n]\right\|} \cdot t_{0}^{2}$ is chi-square distributed, and $\left|t_{3}\right|^{2}$ is exponentially distributed with mean 1. In $\left|t_{2}\right|^{2}$, vector $\mathbf{s}_{i k}[n]$ and the subspace projector $\Pi_{i}^{\perp}[n]$ are independent, but both are orthogonal to $\hat{\mathbf{h}_{i k}}[n]$. Since the independency is conditioned on $\hat{\mathbf{h}_{i k}}[n],\left|t_{2}\right|^{2}$ is beta distributed with Beta $\left(1, N_{t}-2\right)$ and has an average value of $\frac{1}{N_{t}-1}$. Combining these terms together, the average interference is given by

$$
\begin{aligned}
& \mathbb{E}\left\{\left|\mathbf{h}_{i k}[n] \mathbf{w}_{i, a l}[n]\right|^{2}\right\} \\
& =\rho_{c}^{2}\left(2^{-\frac{B}{K\left(N_{t}-1\right)}}\right) \cdot N_{t} \cdot \frac{1}{N_{t}-1}+\left(1-\rho_{c}^{2}\right) \cdot 1 \\
& =1-\rho_{c}^{2}\left(1-\frac{N_{t}}{N_{t}-1} 2^{-\frac{B}{K\left(N_{t}-1\right)}}\right)
\end{aligned}
$$

Note that the average interference power reduces to 0 when both $\rho_{c} \rightarrow 1$ and $B \rightarrow \infty$.

Combining the signal and interference terms and and denoting $\beta_{k}:=\gamma_{k} \sum_{i=1, i \neq k}^{K} \alpha_{i k}^{2}$, the ergodic sum rate lower bounds in (10) can be obtained.

\section{Appendix 2: sum rate using first-order approximation}

In order to derive the sum rate approximation, let us first approximate the two variable function $f(x, y)$ := $\log \left(1+\frac{x}{\frac{1}{\gamma}+y}\right)$ using first-order Taylor series around $\mathbb{E}\{x\}$ and $\mathbb{E}\{y\}$.

$$
\begin{aligned}
f(x, y) \approx & f(\mathbb{E}\{x\}, \mathbb{E}\{y\}) \\
& +f_{x}(\mathbb{E}\{x\}, \mathbb{E}\{y\})(x-\mathbb{E}\{x\}) \\
& +f_{y}(\mathbb{E}\{x\}, \mathbb{E}\{y\})(y-\mathbb{E}\{y\})
\end{aligned}
$$

where $f_{x}(\cdot, \cdot)$ and $f_{y}(\cdot, \cdot)$ are first derivatives with respect to $x$ and $y$, respectively. Applying expectation, the firstorder terms vanish and the following approximation is obtained for $\log \left(1+\frac{x}{\frac{1}{\gamma}+y}\right)$ :

$$
\mathbb{E}\left\{\log \left(1+\frac{x}{\frac{1}{\gamma}+y}\right)\right\} \approx \log \left(1+\frac{\mathbb{E}\{x\}}{\frac{1}{\gamma}+\mathbb{E}\{y\}}\right) .
$$

Such an approximation helps to represent the ergodic sum rates in (8) with an exact Doppler analysis (without high channel correlation assumption).

The average interference powers obtained from Appendix 1 can be directly used in this approximation, so let us re-visit only the average signal powers where Doppler approximations were involved. Instead of the approximate model for $\mathbf{h}_{k k}[n]$ in (13), let us use the model in (3) to express $\mathbf{h}_{k k}[n]$ in terms of $\hat{\mathbf{h}}_{k k}\left[n-D_{f}\right]$, $\mathbf{s}_{k k}\left[n-D_{f}\right]$ and $\mathbf{e}_{k k}[n]$.

Signal power (EgBf) Time shifting by $D_{f}, \mathbb{E}\left\{\mathbf{h}_{k k}[n] \mathbf{w}_{k, e g}\right.$ $\left.\left.[n]\right|^{2}\right\}$ reduces to

$$
\begin{aligned}
\mathbb{E} & \left\{\left|\mathbf{h}_{k k}[n] \mathbf{w}_{k, e g}[n]\right|^{2}\right\} \\
\stackrel{a}{=} & \mathbb{E}\left\{\mid \rho_{l} \sqrt{1-Z_{k k}}\left\|\mathbf{h}_{k k}[n]\right\|+\right. \\
& \left.\left.\sqrt{1-\rho_{l}^{2}} \mathbf{e}_{k k}\left[n+D_{f}\right] \hat{\mathbf{h}}_{k k}^{H}[n]\right|^{2}\right\} \\
= & \rho_{l}^{2} \mathbb{E}\left\{\left\|\mathbf{h}_{k k}[n]\right\|^{2}\right\}\left(1-\mathbb{E}_{C}\left\{Z_{k k}\right\}\right)+ \\
& \left(1-\rho_{l}^{2}\right) \mathbb{E}\left\{\left|\mathbf{e}_{k k}\left[n+D_{f}\right] \hat{\mathbf{h}}_{k k}^{H}[n]\right|^{2}\right\} .
\end{aligned}
$$

(a) is obtained since the quantization error vector is in the null space of $\hat{\mathbf{h}}_{k k}[n] .\left|\mathbf{e}_{k k}\left[n+D_{f}\right] \hat{\mathbf{h}}_{k k}^{H}[n]\right|^{2}$ is an exponentially distributed random variable with mean 1 . Thus, the average signal power reduces to

$$
\begin{aligned}
& \mathbb{E}\left\{\left|\mathbf{h}_{k k}[n] \mathbf{w}_{k, e g}[n]\right|^{2}\right\} \\
& =\rho_{l}^{2} N_{t}\left(1-2^{-\frac{B}{N_{t}-1}}\right)+\left(1-\rho_{l}^{2}\right) \cdot 1 \\
& =\rho_{l}^{2}\left\{N_{t}\left(1-2^{-\frac{B}{N_{t}-1}}\right)-1\right\}+1 .
\end{aligned}
$$

Signal power (AlBf) Using the stationarity of the process to time shift by $D_{f}$,

$$
\begin{aligned}
& \mathbb{E}\left\{\left|\mathbf{h}_{k k}[n] \mathbf{w}_{k, a l}[n]\right|^{2}\right\} \\
& =\mathbb{E}\left\{\left|\rho_{l} \sqrt{1-Z_{k k}} t_{0} t_{1}+\rho_{l} \sqrt{Z_{k k}} t_{0} t_{2}+\sqrt{1-\rho_{l}^{2}} t_{3}\right|^{2}\right\} \\
& =\rho_{l}^{2}\left(1-\mathbb{E}_{C}\left\{Z_{k k}\right\}\right) \mathbb{E}\left\{\left(t_{0} t_{1}\right)^{2}\right\}+ \\
& \rho_{l}^{2} \mathbb{E}_{C}\left\{Z_{k k}\right\} \mathbb{E}\left\{t_{0}^{2}\left|t_{2}\right|^{2}\right\}+\left(1-\rho_{l}^{2}\right) \mathbb{E}\left\{\left|t_{3}\right|^{2}\right\}
\end{aligned}
$$

where $t_{0}:=\left\|\mathbf{h}_{k k}[n]\right\|, t_{1}:=\left\|\mathbf{p}_{k k}[n]\right\|, t_{2}:=\mathbf{s}_{k k}[n] \frac{\mathbf{p}_{k k}[n]}{\left\|\mathbf{p}_{k k}[n]\right\|}$ and $t_{3}:=\mathbf{e}_{k k}\left[n+D_{f}\right] \frac{\Pi_{k}^{\perp}[n] \hat{\mathbf{h}}_{k k}^{H}[n]}{\left\|\Pi_{k}^{\perp}[n] \hat{\mathbf{h}}_{k k}^{H}[n]\right\|}$ with $\mathbf{p}_{k k}[n]$ denoting $\mathbf{p}_{k k}[n]:=\Pi_{k}^{\perp}\left[n-D_{b}\right] \hat{\mathbf{h}}_{k k}^{H}[n]$. For the same reasons as discussed above, the average values of the cross products between these terms are zero.

Let us consider the distribution of the individual terms in $\mathbb{E}\left\{\left|\mathbf{h}_{k k}[n] \mathbf{w}_{k, a l}[n]\right|^{2}\right\} \cdot\left(t_{0} t_{1}\right)^{2}$ is chi-square distributed with $N_{t}-K+1$ degrees of freedom. $\left|t_{3}\right|^{2}$ is exponentially distributed with mean 1 . However, the distribution of the random variable $t_{0}^{2}\left|t_{2}\right|^{2}$ is not obvious due to the orthogonality of $\mathbf{s}_{k k}[n]$ and $\hat{\mathbf{h}}_{k k}[n]$. Nevertheless, the expected 
value of $t_{0}^{2}\left|t_{2}\right|^{2}$ can be determined as follows. Note that the unit vector $\frac{\mathbf{p}_{k k}[n]}{\left\|\mathbf{p}_{k k}[n]\right\|}$ can also be expressed as

$$
\frac{\mathbf{p}_{k k}[n]}{\left\|\mathbf{p}_{k k}[n]\right\|}=\left\|\mathbf{p}_{k k}[n]\right\| \hat{\mathbf{h}}_{k k}[n]+\sqrt{1-\left\|\mathbf{p}_{k k}[n]\right\|^{2}} \mathbf{s}_{k k}^{\prime}[n]
$$

where $\mathbf{s}_{k k}{ }_{k k}[n]$ is another unit vector perpendicular to $\hat{\mathbf{h}}_{k k}[n]$ (in addition to $\mathbf{s}_{k k}[n]$ ). Using this expression and the orthogonality of $\hat{\mathbf{h}}_{k k}[n]$ and $\mathbf{s}_{k k}[n],\left|t_{2}\right|^{2}$ reduces to $\left|t_{2}\right|^{2}=\left(1-t_{1}^{2}\right)\left|\mathbf{s}_{k k}[n] \mathbf{s}_{k k}^{H}[n]\right|^{2}$. Therefore,

$$
\begin{aligned}
& \mathbb{E}\left\{t_{0}^{2}\left|t_{2}\right|^{2}\right\} \\
& \quad=\left(\mathbb{E}\left\{t_{0}^{2}\right\}-\mathbb{E}\left\{\left(t_{0} t_{1}\right)^{2}\right\}\right) \mathbb{E}\left\{\left|\mathbf{s}_{k k}[n] \mathbf{s}_{k k}^{\prime H}[n]\right|^{2}\right\} \\
& =\left(N_{t}-\left(N_{t}-K+1\right)\right) \mathbb{E}\left\{\left|\mathbf{s}_{k k}[n] \mathbf{s}_{k k}^{\prime H}[n]\right|^{2}\right\} .
\end{aligned}
$$

Since $\mathbf{s}_{k k}[n]$ and $\mathbf{s}_{k k}[n]$ are independent and both orthogonal to $\hat{\mathbf{h}}_{k k}[n],\left|\mathbf{s}_{k k}[n] \mathbf{s}_{k k}^{\prime H}[n]\right|^{2}$ has Beta $\left(1, N_{t}-2\right)$ distribution which has an expected value of $\frac{1}{N_{t}-1}$. Thus, the resul to $\mathbb{E}\left\{t_{0}^{2}\left|t_{2}\right|^{2}\right\}=\frac{K-1}{N_{t}-1}$.

Combining the expected values of $\left(t_{0} t_{1}\right)^{2}, t_{0}^{2}\left|t_{2}\right|^{2}$ and $\left|t_{3}\right|^{2}$ obtained above, the average signal power is obtained to be

$$
\begin{aligned}
\mathbb{E} & \left\{\left|\mathbf{h}_{k k}[n] \mathbf{w}_{k, a l}[n]\right|^{2}\right\} \\
= & \rho_{l}^{2}\left(1-2^{-\frac{B}{K\left(N_{t}-1\right)}}\right)\left(N_{t}-K+1\right) \\
& +\rho_{l}^{2}\left(2^{-\frac{B}{K\left(N_{t}-1\right)}}\right) \cdot \frac{K-1}{\left(N_{t}-1\right)}+\left(1-\rho_{l}^{2}\right) \cdot 1 \\
= & \rho_{l}^{2}\left\{\left(N_{t}-K\right)-2^{-\frac{B}{K\left(N_{t}-1\right)}}\left(N_{t}-K+1-\frac{K-1}{N_{t}-1}\right)\right\}+1
\end{aligned}
$$

Combining the average signal and interference powers, the first-order approximations of the ergodic sum rates can be formulated as in (11).

\section{Competing interests}

The authors declare that they have no competing interests.

\section{Author details}

${ }^{1}$ Department of Electronics and Telecommunications, Norwegian University of Science and Technology, Trondheim 7491, Norway. ${ }^{2}$ Mobile Communications Department, EURECOM, Campus SophiaTech, Biot 06410, France.

\section{Received: 12 June 2013 Accepted: 18 October 2013}

\section{Published: 31 October 2013}

\section{References}

1. S Shamai, BM Zaidel, Enhancing the cellular downlink capacity via co-processing at the transmitting end, in Proceedings of the IEEE Vehicular Technology Conference (VTC), (Rhodes, 6-9 May 2001)

2. H Zhang, H Dai, Cochannel interference mitigation cooperative processing in downlink multicell multiuser MIMO networks. EURASIP J. Wireless Commun. Netw. 2004(2), 222-235 (2004). doi:10.1155/S1687147204406148

3. D Gesbert, S Hanly, H Huang, S Shamai Shitz, O Simeone, W Yu, Multi-cell MIMO cooperative networks: a new look at interference. IEEE J. Sel. Areas Commun. 28(9), 1380-1408 (2010). doi:10.1109/JSAC.2010.101202
4. S Jing, DNC Tse, JB Soriaga, J Hou, JE Smee, R Padovani, Multicell downlink capacity with coordinated processing. EURASIP J. Wireless Commun. Netw. 2008, 18-11819 (2008). doi:10.1155/2008/586878

5. F Boccardi, B Clerckx, A Ghosh, E Hardouin, G Jö andngren, K Kusume, E Onggosanusi, Y Tang, Multiple-antenna techniques in LTE-Advanced. IEEE Commun. Lett. 50(3), 114-121 (2012). doi:10.1109/MCOM.2012.6163590

6. IF Akyildiz, DM Gutierrez-Estevez, EC Reyes, The evolution to 4G cellular systems: LTE-Advanced. Phys. Commun. 3(4), 217-244 (2010). doi:10.1016/j.phycom.2010.08.001

7. A Papadogiannis, HJ Bang, D Gesbert, E Hardouin, Downlink overhead reduction for multi-cell cooperative processing enabled wireless networks, in Proceedings of the IEEE International Symposium on Personal, Indoor and Mobile Radio Communications (PIMRC), (Cannes, 15-18 Sept 2008)

8. O Simeone, O Somekh, HV Poor, S Shamai, Downlink multicell processing with limited-backhaul capacity. EURASIP J. Adv. Signal Process. 2009, 3-1310 (2009). doi:10.1155/2009/840814

9. D Samardzija, H Huang, Determining backhaul bandwidth requirements for network MIMO, in Proceedings of the European Signal Processing Conference (EUSIPCO), (Glasgow, 24-28 Aug 2009)

10. N Seifi, M Viberg, RW Heath, J Zhang, M Coldrey, Coordinated single-cell vs multi-cell transmission with limited-capacity backhaul, in Proceedings of the Asilomar Conference on Signals, Systems and Computers, (Pacific Grove, 7-10 Nov 2010)

11. E Larsson, E Jorswieck, Competition versus cooperation on the MISO interference channel. IEEE J. Sel. Areas Commun. 26(7), 1059-1069 (2008). doi:10.1109/JSAC.2008.080904

12. EA Jorswieck, EG Larsson, D Danev, Complete characterization of the pareto boundary for the MISO interference channel. IEEE Trans. Signal Process. 56(10), 5292-5296 (2008). doi:10.1109/TSP.2008.928095

13. J Lindblom, E Karipidis, EG Larsson, Selfishness and altruism on the MISO interference channel: the case of partial transmitter CSI. IEEE Commun. Lett. 13(9), 667-669 (2009). doi:10.1109/LCOMM.2009.090970

14. J Zhang, RW Heath, M Kountouris, JG Andrews, Mode switching for the multi-antenna broadcast channel based on delay and channel quantization. EURASIP J. Adv. Signal Process. 2009, 1-1115 (2009). doi:10.1155/2009/802548

15. R Fritzsche, GP Fettweis, CSI distribution for joint processing in cooperative cellular networks, in Proceedings of the IEEE Vehicula Technology Conference (VTC), (San Francisco, 5-8 Sept 2011)

16. R Bhagavatula, RW Heath, Adaptive bit partitioning for multicell intercell interference nulling with delayed limited feedback. IEEE Trans. Signal Process. 59(8), 3824-3836 (2011). doi:10.1109/TSP.2011.2146777

17. DJ Love, RW Heath, VKN Lau, D Gesbert, BD Rao, M Andrews, An overview of limited feedback in wireless communication systems. IEEE J. Sel. Areas Commun. 26(8), 1341-1365 (2008). doi:10.1109/JSAC.2008.081002

18. N Jindal, MIMO broadcast channels with finite-rate feedback. IEEE Trans. Inf. Theory 52(11), 5045-5060 (2006). doi:10.1109/TIT.2006.883550

19. J Zhang, JG Andrews, Adaptive spatial intercell interference cancellation in multicell wireless networks. IEEE J. Sel. Areas Commun. 28(9), 1455-1468 (2010). doi:10.1109/JSAC.2010.101207

20. RH Clark, A statistical theory of mobile radio reception. Bell Syst. Tech. J. 47(2), 957-1000 (1968)

21. CC Tan, NC Beaulieu, On first-order Markov modeling for the Rayleigh fading channel. IEEE Trans. Commun. 48(12), 2032-2040 (2000). doi:10.1109/26.891214

22. W Santipach, ML Honig, Asymptotic capacity of beamforming with limited feedback, in Proceedings of the International Symposium on Information Theory (ISIT), (Chicago, 27 June-2 July 2004)

23. R Zakhour, D Gesbert, Coordination on the MISO interference channel using the virtual SINR framework, in Proceedings of the Intenational Workshop on Smart Antennas (WSA), (Berlin, 16-18 Feb 2009)

24. S He, Y Huang, L Yang, A Nallanathan, P Liu, A multi-cell beamforming design by uplink-downlink max-min SINR duality. IEEE Trans. Wireless Commun. 11(8), 2858-2867 (2012). doi:10.1109/TWC.2012.061912.111553

25. BL Ng, JS Evans, SV Hanly, D Aktas, Distributed downlink beamforming with cooperative base stations. IEEE Trans. Inf. Theory 54(12), 5491-5499 (2008). doi:10.1109/TIT.2008.2006426 
26. A Ekbal, JM Cioffi, Distributed transmit beamforming in cellular networks a convex optimization perspective, in Proceedings of the IEEE International Conference on Communications (ICC), (Seoul, 16-20 May 2005)

27. R Bhagavatula, RW Heath, B Rao, Limited feedback with joint CSI quantization for multicell cooperative generalized eigenvector beamforming, in Proceedings of the IEEE International Conference on Acoustics, Speech and Signal Processing (ICASSP), (Dallas, 14-19 Mar 2010)

doi:10.1186/1687-1499-2013-253

Cite this article as: Godana and Gesbert: Egoistic vs. altruistic beamforming in multi-cell systems with feedback and back-haul delays. EURASIP Journal on Wireless Communications and Networking 2013 2013:253.

\section{Submit your manuscript to a SpringerOpen ${ }^{\circ}$ journal and benefit from:}

- Convenient online submission

- Rigorous peer review

- Immediate publication on acceptance

- Open access: articles freely available online

- High visibility within the field

- Retaining the copyright to your article

Submit your next manuscript at $\boldsymbol{\triangleright}$ springeropen.com 